\title{
The Backward Euler Fully Discrete Finite Volume Method for the Problem of Purely Longitudinal Motion of a Homogeneous Bar
}

\section{Ziwen Jiang and Deren Xie}

School of Mathematical Sciences, Shandong Normal University, Jinan, Shandong 250014, China

Correspondence should be addressed to Ziwen Jiang, ziwenjiang@163.com

Received 28 September 2012; Accepted 29 November 2012

Academic Editor: Xiaodi Li

Copyright (C) 2012 Z. Jiang and D. Xie. This is an open access article distributed under the Creative Commons Attribution License, which permits unrestricted use, distribution, and reproduction in any medium, provided the original work is properly cited.

We present a linear backward Euler fully discrete finite volume method for the initial-boundaryvalue problem of purely longitudinal motion of a homogeneous bar and an give optimal order error estimates in $L^{2}$ and $H^{1}$ norms. Furthermore, we obtain the superconvergence error estimate of the generalized projection of the solution $u$ in $H^{1}$ norm. Numerical experiment illustrates the convergence and stability of this scheme.

\section{Introduction}

We consider the following mixed boundary-initial value problem:

$$
\begin{aligned}
& \text { (a) } u_{t t}=u_{x x t}+f\left(u_{x}\right)_{x}, \quad(x, t) \in(0,1) \times[0, T], \\
& \text { (b) } u(x, 0)=u_{0}(x), \quad u_{t}(x, 0)=u_{1}(x), \quad x \in(0,1), \\
& \text { (c) } u(0, t)=u(1, t)=0, \quad t \in[0, T] .
\end{aligned}
$$

This problem (1.1) arises when one considers the purely longitudinal motion of a homogeneous bar [1]. The displacement of the cross-section of the bar at time $t$ is denoted by $u(x, t)$. When both ends of the bar are fixed, $u(0, t)=u(1, t)=0, t \in[0, T]$, that is the boundary condition. $u(x, 0)=u_{0}(x)$ and $u_{t}(x, 0)=u_{1}(x)$ are the initial data.

In theoretical analysis, the problem (1.1) was first treated by Greenberg et al. [2], by assuming that the function $f$ was monotonic, that is,

$$
f^{\prime}(s)>0, \quad \forall s \in(-\infty,+\infty),
$$


and that the initial data was smooth, specifically

$$
u_{0} \in C^{4}([0,1]), \quad u_{1} \in C^{2}([0,1]) .
$$

Under these assumptions they showed the existence of a unique smooth solution which decays the zero solution as $t \rightarrow \infty$. (See also Greenberg [3] and Greenberg and MacCamy [4].) Andrews [1] made the hypotheses that $u_{0} \in W_{0}^{1, \infty}(0,1), u_{1} \in H_{0}^{1}(0,1)$, the function $f$ is locally Lipschitz continuous, and there exists a constant $a_{0}>0$ such that $\left(f\left(s_{1}\right)-f\left(s_{2}\right)\right)\left(s_{1}-\right.$ $\left.s_{2}\right)>0$ whenever $\left|s_{1}-s_{2}\right| \geq a_{0}$; then he proved the existence of a unique global weak solution. Under the hypotheses that $u_{0}, u_{1} \in H^{2}(0,1) \cap H_{0}^{1}(0,1)$, and there exists a constant $a_{1}>0$ such that $f^{\prime}(s) \geq a_{1}, s \in(-\infty,+\infty)$, Y. Liu and D. Liu [5] proved the existence of a unique global strong solution of the problem (1.1).

Numerical simulation methods for the problem (1.1) are recently studied by several authors ([6-11]). In [6], Gao et al. studied a finite difference method of the problem (1.1) in the domain $[0,1] \times[0, T](T>0)$ and proved the convergence of the method by using discrete functional analysis and prior estimate. In [7, 8], Jiang et al. studied two finite element methods of (1.1) and obtained the optimal error estimates of this finite element scheme in $L^{2}$ and $H^{1}$ norms. In [9], Z. Jiang and Y. Jiang, and in [10], Jiang and Li, studied a mixed finite element method and a expanded mixed finite element method, respectively, and obtained the optimal error estimates of these schemes. However, few work on finite volume methods of (1.1) was found (see [11]). As we know, finite volume methods (also called generalized difference methods) were proposed in eighties last century and developed very quickly. Now this kind of method becomes one of the main numerical methods for solving differential equations, for example, convection diffusion equations [12-14] and Navier-Stokes equations [15].

In this paper we want to make further study of finite volume methods for the problem (1.1). First in Section 2, we derive a finite volume weak form of (1.1) in the case that $f(s)$ is nonlinear, then propose a linear backward Euler fully discrete finite volume scheme of (1.1). Existence and uniqueness of the solution of this scheme are proved. Next, in Section 3, we give optimal error estimates in $L^{2}$ and $H^{1}$ norms and superconvergence in $H^{1}$ norm by using new defined projections. Numerical experiments and computational results are presented in Section 4, which confirm our theoretical analysis.

\section{The Linear Backward Euler Fully Discrete Finite Volume Scheme}

In this section, we construct the finite volume method of the problem (1.1) and prove the existence and uniqueness of the solution of this finite volume scheme.

Firstly, let $T_{h}$ be a partition for the interval $I=[0,1]$, with its nodes $0=x_{0}<x_{1}<\cdots<$ $x_{r}=1$. The length of the element $I_{i}=\left[x_{i-1}, x_{i}\right]$ is denoted by $h_{i}=x_{i}-x_{i-1}, i=1,2, \ldots, r, h=$ $\max _{1 \leq i \leq r} h_{i}$ is maximum of $h_{i}$. We suppose $T_{h}$ is regular, that is, there exists a positive constant $\mu>0$ such that $h_{i} \geq \mu h, i=1,2, \ldots, r$. For the definition of the finite volume scheme, the dual partition $T_{h}^{*}$ of $T_{h}$ is needed, which is $0=x_{0}<x_{1 / 2}<x_{3 / 2}<\cdots<x_{r-1 / 2}<x_{r}=1$. The dual elements are denoted by $I_{0}^{*}=\left[x_{0}, x_{1 / 2}\right], I_{j}^{*}=\left[x_{j-1 / 2}, x_{j+1 / 2}\right], j=1,2, \ldots, r-1, I_{r}^{*}=\left[x_{r-1 / 2}, x_{r}\right]$, where $x_{j-1 / 2}=(1 / 2)\left(x_{j-1}+x_{j}\right), j=1,2, \ldots, r$.

Secondly, we define the piecewise linear trial function space $U_{h}$ over the partition $T_{h}$, satisfying $U_{h} \subset H_{0}^{1}(I)$, where $H_{0}^{1}(I)$ is the Sobolev space on $I$. Then $u_{h}(x)=\sum_{i=1}^{r-1} u_{h}\left(x_{i}\right) \varphi_{i}(x)$ 
for all $u_{h}(x) \in U_{h}$, where $\varphi_{i}(x)$ is the basis function associated with the nodes $x_{i}(i=$ $1,2, \ldots, r-1)$,

$$
\varphi_{i}(x)= \begin{cases}1-\frac{x_{i}-x}{h_{i}}, & x \in I_{i}, \\ 1-\frac{x-x_{i}}{h_{i+1}}, & x \in I_{i+1}, \\ 0, & x \notin I_{i} \cup I_{i+1} .\end{cases}
$$

It is easy to know that the derivative of $u_{h}$ with respect to $x$ is

$$
u_{h x}(x)=\frac{u_{h}\left(x_{i}\right)-u_{h}\left(x_{i-1}\right)}{h_{i}}, \quad x_{i-1} \leq x \leq x_{i}, i=1,2, \ldots, r
$$

The test function space $V_{h} \subset L^{2}(I)$ associated with the dual partition $T_{h}^{*}$ is defined as the set of all piecewise constants with $v_{h}(0)=v_{h}(1)=0$ for all $v_{h}(x) \in V_{h}$. We may choose the basis function $\psi_{j}(x)$ of $V_{h}$ in such a way that $\psi_{j}(x)$ is the characteristic function of $I_{j}^{*}$, that is,

$$
\psi_{j}(x)=\left\{\begin{array}{ll}
1, & x \in I_{j}^{*} \\
0, & x \notin I_{j}^{*},
\end{array} \quad j=1,2, \ldots, r-1\right.
$$

Then for any $v_{h}(x) \in V_{h}$ can be expressed as $v_{h}(x)=\sum_{j=1}^{r-1} v_{h}\left(x_{j}\right) \psi_{j}(x)$.

Obviously,

$$
\begin{gathered}
U_{h} \subset H_{0}^{1}(I), \quad V_{h} \subset L^{2}(I), \\
\operatorname{dim} U_{h}=\operatorname{dim} V_{h}=r-1 .
\end{gathered}
$$

Meanwhile, $U_{h} \subset W_{0}^{1, \infty}(I)$.

Thirdly, for the time interval $[0, T]$, we give an isometric partition and denote the nodes $t_{i}=i \tau, i=0,1, \ldots, N, \tau=T / N$.

We introduce some notations for functions $u(x, t)$ and $f\left(u_{x}(x, t)\right)$ :

$$
\begin{gathered}
u^{n}=u\left(x, t_{n}\right), \quad u_{j}=u\left(x_{j}, t\right), \quad u_{j}^{n}=u\left(x_{j}, t_{n}\right), \quad \partial_{t} u^{n}=\frac{u^{n+1}-u^{n}}{\tau}, \\
\partial_{t t}^{2} u^{n}=\frac{u^{n+1}-2 u^{n}+u^{n-1}}{\tau^{2}}, \quad u^{n+1 / 2}=\frac{u^{n+1}+u^{n}}{2} \\
u^{n, 1 / 4}=\frac{u^{n+1}+2 u^{n}+u^{n-1}}{4}, \quad f^{1 / 2}\left(u_{x}^{n}\right)=\frac{f\left(u_{x}^{n+1}\right)+f\left(u_{x}^{n}\right)}{2}, \\
f^{1 / 4}\left(u_{x}^{n}\right)=\frac{f\left(u_{x}^{n+1}\right)+2 f\left(u_{x}^{n}\right)+f\left(u_{x}^{n-1}\right)}{4}, \quad f^{(1 / 4) *}\left(u_{x}^{n}\right)=\frac{3 f\left(u_{x}^{n}\right)+f\left(u_{x}^{n-1}\right)}{4} .
\end{gathered}
$$


Then we can get

$$
\begin{gathered}
\partial_{t t}^{2} u^{n}=\frac{\partial_{t} u^{n}-\partial_{t} u^{n-1}}{\tau}, \quad u^{n, 1 / 4}=\frac{u^{n+1 / 2}+u^{n-1 / 2}}{2}, \\
\frac{1}{2}\left(\partial_{t} u^{n}+\partial_{t} u^{n-1}\right)=\frac{1}{\tau}\left(u^{n+1 / 2}-u^{n-1 / 2}\right) .
\end{gathered}
$$

Let $u$ be the solution of (1.1). Integrating (1.1) a over the dual element $I_{j}^{*} \in T_{h^{\prime}}^{*}$ we obtain

$$
\int_{x_{j-1 / 2}}^{x_{j+1 / 2}} u_{t t} d x+u_{x t}\left(x_{j-1 / 2}\right)-u_{x t}\left(x_{j+1 / 2}\right)+f\left(u_{x}\left(x_{j-1 / 2}\right)\right)-f\left(u_{x}\left(x_{j+1 / 2}\right)\right)=0
$$

where $j=0,1, \ldots, r, x_{-1 / 2}=x_{0}, x_{r+1 / 2}=x_{r}$, and $0<t \leq T$. The problem (2.7) can be rewritten in a variational form. For any arbitrary $v_{h} \in V_{h}$, we multiply the integral relation in (2.7) by $v_{h}\left(x_{j}\right)$ and sum over all $j=0,1, \ldots, r$ to obtain

$$
\begin{gathered}
\left(u_{t t}, v_{h}\right)+a^{*}\left(u_{t}, v_{h}\right)+b^{*}\left(f(u), v_{h}\right)=0, \quad \forall v_{h} \in V_{h}, t \in(0, T] \\
u(0)=u_{0}, \quad u_{t}(0)=u_{1}
\end{gathered}
$$

where for any arbitrary $w=\sum_{j=1}^{r-1} w_{j} \psi_{j} \in V_{h}$, the bilinear forms $a^{*}(v, w), b^{*}(f(v), w)$ are defined by

$$
\begin{gathered}
a^{*}(v, w)=\sum_{j=1}^{r-1} w_{j} a^{*}\left(v, \psi_{j}\right), \quad a^{*}\left(v, \psi_{j}\right)=v_{x}\left(x_{j-1 / 2}\right)-v_{x}\left(x_{j+1 / 2}\right), \\
b^{*}(f(v), w)=\sum_{j=1}^{r-1} w_{j} b^{*}\left(f(v), \psi_{j}\right), \quad b^{*}\left(f(v), \psi_{j}\right)=f\left(v_{x}\left(x_{j-1 / 2}\right)\right)-f\left(v_{x}\left(x_{j+1 / 2}\right)\right) .
\end{gathered}
$$

Since $f$ is a nonlinear function, we will consider the following linear finite volume scheme: find $u_{h}^{n} \in U_{h}, n=0,1,2, \ldots, N$ such that

$$
\begin{gathered}
\left(\partial_{t t}^{2} u_{h^{\prime}}^{n} v_{h}\right)+a^{*}\left(\partial_{t} u_{h^{\prime}}^{n} v_{h}\right)+b^{*}\left(f^{(1 / 4) *}\left(u_{h}^{n}\right), v_{h}\right)=0, \quad \forall v_{h} \in V_{h}, n=1, \ldots, N-1, \\
u_{h}^{0}=\prod_{h} u_{0}, \quad u_{h}^{1}=\Pi_{h} u_{0}+\tau \Pi_{h} u_{1},
\end{gathered}
$$

where $\Pi_{h} u_{0}$ and $\Pi_{h} u_{1}$ are the interpolation projection of $u_{0}$ and $u_{1}$ onto the trial function space $U_{h}$, respectively, and the interpolation operator $\Pi_{h}$ is defined as $\Pi_{h}: H_{0}^{1}(I) \rightarrow U_{h}$

$$
\Pi_{h} w=\sum_{i=1}^{r-1} w_{i} \varphi_{i}, \quad \forall w \in H_{0}^{1}(I)
$$


We also need to introduce the interpolation $\Pi_{h}^{*}: H_{0}^{1}(I) \rightarrow V_{h}$, defined by,

$$
\Pi_{h}^{*} w=\sum_{i=1}^{r-1} w_{i} \psi_{i}, \quad \forall w \in H_{0}^{1}(I)
$$

By Sobolev's interpolation theory, we know that

$$
\left|w-\Pi_{h} w\right|_{m, p} \leq c h^{2-m}|w|_{2, p^{\prime}} \quad m=0,1,1 \leq p \leq \infty
$$

In this paper we adopt the standard notation $W^{m, p}(I)$ for Sobolev space on $I$ with norm $\|\cdot\|_{m, p}$ and seminorm $|\cdot|_{m, p}$. In order to simplify the notations, we denote $W^{m, 2}(I)$ by $H^{m}(I)$ and skip the index $p=2$ when possible, that is, $|\cdot|_{m, p}=|\cdot|_{m},\|\cdot\|_{m, p}=\|\cdot\|_{m},\|\cdot\|_{0}=\|\cdot\|$. We denote by $L^{q}\left(0, T ; W^{m, p}(I)\right)$ the Banach space of all $L^{q}$ integrable functions from $(0, T)$ into $W^{m, p}(I)$ with the norm $\|\cdot\|_{L^{q}\left(W^{m, p}\right)}=\left(\int_{0}^{T}\|\cdot\|_{m, p}^{q} d t\right)^{1 / q}$ for $q \in[1, \infty)$ and the standard modification for $q=\infty$. After all these denotations, we give the existence and uniqueness of the solution of the finite volume scheme (2.10).

Theorem 2.1. The solution of the finite volume scheme (2.10) is existent and unique.

Proof. Let $u_{h}^{n}=\sum_{i=1}^{r-1} u_{h i}^{n} \varphi_{i}(x) \in U_{h}(n=0,1,2, \ldots, N)$ be the solution of (2.10). According to $u_{h}^{0}=\Pi_{h} u_{0}$ and $u_{h}^{1}=\Pi_{h} u_{0}+\tau \prod_{h} u_{1}, u_{h j}^{0}=u_{0}\left(x_{j}\right), u_{h j}^{1}=u_{0}\left(x_{j}\right)+\tau u_{1}\left(x_{j}\right), j=1,2, \ldots, r-1$ are known. Hence the existence and uniqueness of the solution $u_{h}^{n}(n=0,1,2, \ldots, N)$ of scheme (2.10) are equivalent to the existence and uniqueness of $\left\{u_{h i}^{n}\right\}_{i=1}^{r-1}, n=2, \ldots, N$.

For $n=2, \ldots, N$, choosing $v_{h}=\psi_{j}, j=1,2, \ldots, r-1$. By (2.2) we have

$$
\begin{aligned}
\left(\partial_{t t}^{2} u_{h}^{n-1}, v_{h}\right)= & \int_{0}^{1} \partial_{t t}^{2} u_{h}^{n-1} \psi_{j}(x) d x=\int_{x_{j-1 / 2}}^{x_{j+1 / 2}} \partial_{t t}^{2} u_{h}^{n-1} d x \\
= & \frac{1}{\tau^{2}} \int_{x_{j-1 / 2}}^{x_{j+1 / 2}}\left(u_{h}^{n}-2 u_{h}^{n-1}+u_{h}^{n-2}\right) d x \\
= & \frac{1}{\tau^{2}} \int_{x_{j-1 / 2}}^{x_{j+1 / 2}} \sum_{i=1}^{r-1}\left(u_{h i}^{n}-2 u_{h i}^{n-1}+u_{h i}^{n-2}\right) \varphi_{i}(x) d x \\
= & \frac{1}{\tau^{2}}\left\{\left(u_{h j-1}^{n}-2 u_{h j-1}^{n-1}+u_{h j-1}^{n-2}\right) \int_{x_{j-1 / 2}}^{x_{j+1 / 2}} \varphi_{j-1}(x) d x\right. \\
& +\left(u_{h j}^{n}-2 u_{h j}^{n-1}+u_{h j}^{n-2}\right) \int_{x_{j-1 / 2}}^{x_{j+1 / 2}} \varphi_{j}(x) d x \\
& \left.+\left(u_{h j+1}^{n}-2 u_{h j+1}^{n-1}+u_{h j+1}^{n-2}\right) \int_{x_{j-1 / 2}}^{x_{j+1 / 2}} \varphi_{j+1}(x) d x\right\}
\end{aligned}
$$




$$
\begin{aligned}
& =\frac{1}{\tau^{2}}\left\{\frac{1}{8} h_{j}\left(u_{h j-1}^{n}-2 u_{h j-1}^{n-1}+u_{h j-1}^{n-2}\right)\right. \\
& +\frac{3}{8}\left(h_{j}+h_{j+1}\right)\left(u_{h j}^{n}-2 u_{h j}^{n-1}+u_{h j}^{n-2}\right) \\
& \left.+\frac{1}{8} h_{j+1}\left(u_{h j+1}^{n}-2 u_{h j+1}^{n-1}+u_{h j+1}^{n-2}\right)\right\} \text {, } \\
& a^{*}\left(\partial_{t} u_{h}^{n-1}, v_{h}\right)=a^{*}\left(\partial_{t} u_{h}^{n-1}, \psi_{j}\right)=\partial_{t} u_{h x}^{n-1}\left(x_{j-1 / 2}\right)-\partial_{t} u_{h x}^{n-1}\left(x_{j+1 / 2}\right) \\
& =\partial_{t} \frac{u_{h j}^{n-1}-u_{h j-1}^{n-1}}{h_{j}}-\partial_{t} \frac{u_{h j+1}^{n-1}-u_{h j}^{n-1}}{h_{j+1}} \\
& =\frac{1}{\tau h_{j}}\left(u_{h j}^{n}-u_{h j-1}^{n}-u_{h j}^{n-1}+u_{h j-1}^{n-1}\right) \\
& -\frac{1}{\tau h_{j+1}}\left(u_{h j+1}^{n}-u_{h j}^{n}-u_{h j+1}^{n-1}+u_{h j}^{n-1}\right), \\
& b^{*}\left(f^{(1 / 4) *}\left(u_{h}^{n-1}\right), v_{h}\right)=b^{*}\left(f^{(1 / 4) *}\left(u_{h}^{n-1}\right), \psi_{j}\right) \\
& =f^{(1 / 4) *}\left(u_{h x}^{n-1}\left(x_{j-1 / 2}\right)\right)-f^{(1 / 4) *}\left(u_{h x}^{n-1}\left(x_{j+1 / 2}\right)\right) \\
& =f^{(1 / 4) *}\left(\frac{u_{h j}^{n-1}-u_{h j-1}^{n-1}}{h_{j}}\right)-f^{(1 / 4) *}\left(\frac{u_{h j+1}^{n-1}-u_{h j}^{n-1}}{h_{j+1}}\right) \\
& =\frac{3}{4} f\left(\frac{u_{h j}^{n-1}-u_{h j-1}^{n-1}}{h_{j}}\right)+\frac{1}{4} f\left(\frac{u_{h j}^{n-2}-u_{h j-1}^{n-2}}{h_{j}}\right) \\
& -\frac{3}{4} f\left(\frac{u_{h j+1}^{n-1}-u_{h j}^{n-1}}{h_{j+1}}\right)-\frac{1}{4} f\left(\frac{u_{h j+1}^{n-2}-u_{h j}^{n-2}}{h_{j+1}}\right) \text {. }
\end{aligned}
$$

Using (2.10) we obtain

$$
\begin{aligned}
\frac{1}{\tau^{2}}\left[\frac{1}{8} h_{j}\left(u_{h j-1}^{n}-2 u_{h j-1}^{n-1}+u_{h j-1}^{n-2}\right)+\frac{3}{8}\left(h_{j}+h_{j+1}\right)\left(u_{h j}^{n}-2 u_{h j}^{n-1}+u_{h j}^{n-2}\right)\right. \\
\left.+\frac{1}{8} h_{j+1}\left(u_{h j+1}^{n}-2 u_{h j+1}^{n-1}+u_{h j+1}^{n-2}\right)\right] \\
+\frac{1}{\tau h_{j}}\left(u_{h j}^{n}-u_{h j-1}^{n}-u_{h j}^{n-1}+u_{h j-1}^{n-1}\right) \\
-\frac{1}{\tau h_{j+1}}\left(u_{h j+1}^{n}-u_{h j}^{n}-u_{h j+1}^{n-1}+u_{h j}^{n-1}\right)
\end{aligned}
$$


Abstract and Applied Analysis

$$
\begin{aligned}
& +\frac{3}{4} f\left(\frac{u_{h j}^{n-1}-u_{h j-1}^{n-1}}{h_{j}}\right)+\frac{1}{4} f\left(\frac{u_{h j}^{n-2}-u_{h j-1}^{n-2}}{h_{j}}\right) \\
& -\frac{3}{4} f\left(\frac{u_{h j+1}^{n-1}-u_{h j}^{n-1}}{h_{j+1}}\right)-\frac{1}{4} f\left(\frac{u_{h j+1}^{n-2}-u_{h j}^{n-2}}{h_{j+1}}\right)=0,
\end{aligned}
$$

that is,

$$
\begin{aligned}
\left(\frac{h_{j}}{8}-\right. & \left.\frac{\tau}{h_{j}}\right) u_{h j-1}^{n}+\left[\frac{3}{8}\left(h_{j}+h_{j+1}\right)+\tau\left(\frac{1}{h_{j}}+\frac{1}{h_{j+1}}\right)\right] u_{h j}^{n}+\left(\frac{h_{j+1}}{8}-\frac{\tau}{h_{j+1}}\right) u_{h j+1}^{n} \\
= & \left(\frac{h_{j}}{4}-\frac{\tau}{h_{j}}\right) u_{h j-1}^{n-1}+\left[\frac{3}{4}\left(h_{j}+h_{j+1}\right)+\tau\left(\frac{1}{h_{j}}+\frac{1}{h_{j+1}}\right)\right] u_{h j}^{n-1} \\
& +\left(\frac{h_{j+1}}{4}-\frac{\tau}{h_{j+1}}\right) u_{h j+1}^{n-1}+\frac{3 \tau^{2}}{4}\left[f\left(\frac{u_{h j+1}^{n-1}-u_{h j}^{n-1}}{h_{j+1}}\right)-f\left(\frac{u_{h j}^{n-1}-u_{h j-1}^{n-1}}{h_{j}}\right)\right] \\
& -\frac{1}{8} h_{j} u_{h j-1}^{n-2}-\frac{3}{8}\left(h_{j}+h_{j+1}\right) u_{h j}^{n-2}-\frac{1}{8} h_{j+1} u_{h j+1}^{n-2} \\
& +\frac{\tau^{2}}{4}\left[f\left(\frac{u_{h j+1}^{n-2}-u_{h j}^{n-2}}{h_{j+1}}\right)-f\left(\frac{u_{h j}^{n-2}-u_{h j-1}^{n-2}}{h_{j}}\right)\right], \quad j=1,2, \ldots, r-1 .
\end{aligned}
$$

Noticing $u_{h 0}^{n}=u_{h r}^{n}=0$, the coefficient matrix of the system (2.16) is strictly diagonally dominant matrix. So, when $\left\{u_{h i}^{n-1}\right\}_{i=1}^{r-1}$ and $\left\{u_{h i}^{n-2}\right\}_{i=1}^{r-1}$ are known, the solution $\left\{u_{h i}^{n}\right\}_{i=1}^{r-1}$ is existent and unique. Combining the above conditions, the solution $\left\{u_{h i}^{n}\right\}_{i=1}^{r-1}(n=2, \ldots, N)$ is existent and unique when $\left\{u_{h i}^{0}\right\}_{i=1}^{r-1}$ and $\left\{u_{h i}^{1}\right\}_{i=1}^{r-1}$ are known. This completes the proof.

\section{Error Estimates}

In this section, we will prove the optimal error estimates in the $L^{2}$ and $H^{1}$ norms as well as the superconvergence error estimates in the $H^{1}$ norm. This needs some assumptions about the data.

$\left(\mathrm{H}_{1}\right)$ The nonlinear function $f(s)$ satisfies

$$
0<l \leq f^{\prime}(s) \leq L, \quad \max \left\{\left|f^{\prime \prime}(s)\right|,\left|f^{\prime \prime \prime}(s)\right|\right\} \leq M, \quad s \in(-\infty,+\infty) .
$$

$\left(\mathrm{H}_{2}\right)$ The initial functions $u_{0}$ and $u_{1}$ satisfy

$$
u_{0} \in H_{0}^{1}(I) \cap H^{2}(I), \quad u_{1} \in H_{0}^{1}(I) \cap W^{3,1}(I) .
$$


$\left(\mathrm{H}_{3}\right)$ The solution $u$ of $(1.1)$ satisfies

$$
\begin{gathered}
u \in L^{\infty}\left(0, T ; W^{3,1}(I)\right), \quad u_{t} \in L^{\infty}\left(0, T ; W^{2, \infty}(I) \cap W^{3,1}(I)\right), \\
u_{t t} \in L^{\infty}\left(0, T ; W^{3,1}(I)\right), \quad u_{t t t t} \in L^{2}\left(0, T ; L^{2}(I)\right) .
\end{gathered}
$$

For any $u_{h}, v_{h} \in U_{h}, u_{h}=\sum_{i=1}^{r-1} u_{h i} \varphi_{i}(x), v_{h}=\sum_{i=1}^{r-1} v_{h i} \varphi_{i}(x)$, where $u_{h i}=u_{h}\left(x_{i}\right), v_{h i}=$ $v_{h}\left(x_{i}\right), i=1,2, \ldots, r-1$. Using the definition of the interpolation operator $\Pi_{h}^{*}$ we know that $\Pi_{h}^{*} u_{h}=\sum_{i=1}^{r-1} u_{h i} \varphi_{i}(x), \Pi_{h}^{*} v_{h}=\sum_{i=1}^{r-1} v_{h i} \varphi_{i}(x) \in V_{h}$. Noting $u_{h 0}=u_{h r}=v_{h 0}=v_{h r}=0$ and [11, Lemma 3.1] we have

$$
\left(u_{h}, \Pi_{h}^{*} v_{h}\right)=\left(v_{h}, \Pi_{h}^{*} u_{h}\right)=\frac{1}{8} \sum_{i=1}^{r-1}\left[h_{i} u_{h i-1} v_{h i}+3\left(h_{i}+h_{i+1}\right) u_{h i} v_{h i}+h_{i+1} u_{h i+1} v_{h i}\right]
$$

Hence

$$
\left(u_{h}, \Pi_{h}^{*} u_{h}\right)=\frac{1}{8} \sum_{i=1}^{r-1}\left[h_{i}\left(u_{h i}+u_{h i+1}\right)^{2}+2\left(h_{i}+h_{i+1}\right) u_{h i}^{2}\right]+h_{1} u_{h 1}^{2}
$$

So, when $u_{h} \neq 0$, we can get $\left(u_{h}, \Pi_{h}^{*} u_{h}\right)>0$. Let $\mid\left\|u_{h}\right\|_{0}=\left(u_{h}, \Pi_{h}^{*} u_{h}\right)^{1 / 2}$. By [11, Lemma 3.2], we have

$$
\sqrt{\frac{2}{3}}\left|\left\|u_{h}\right\|\right|_{0} \leq\left\|u_{h}\right\| \leq \sqrt{\frac{4}{3}}\left|\left\|u_{h}\right\|\right|_{0}, \quad \forall u_{h} \in U_{h} .
$$

On the other hand, using [11, Lemma 3.3], we also have

$$
a^{*}\left(u_{h}, \Pi_{h}^{*} u_{h}\right)=\sum_{i=1}^{r} \frac{1}{h_{i}}\left(u_{h i}-u_{h i-1}\right)^{2}=\left|u_{h}\right|_{1}^{2}
$$

For error estimate, we will use the generalized adjoint finite volume element projection $\widetilde{u}^{[11]}$ of the solution $u$ of (1.1), that is, $\tilde{u}:[0, T] \rightarrow U_{h}$ satisfies

$$
\begin{gathered}
a^{*}\left(u_{t}-\tilde{u}_{t}, v_{h}\right)+b^{*}\left(f(u)-f(\tilde{u}), v_{h}\right)=0, \quad \forall v_{h} \in V_{h}, 0<t \leq T, \\
\tilde{u}(0)=\Pi_{h} u_{0} .
\end{gathered}
$$

Let $u$ and $\tilde{u}$ denote the solutions of (1.1) and (3.8), respectively. Under the assumptions $\left(\mathrm{H}_{1}\right)$, $\left(\mathrm{H}_{2}\right)$, and $\left(\mathrm{H}_{3}\right)$ together with [11, Theorems 3.4-3.5] and the one-dimensional imbedding theorem in Sobolev space, then we can obtain the following. 
Lemma 3.1. Let $u$ and $\tilde{u}$ be the solutions of (1.1) and (3.8), respectively. If the assumptions $\left(H_{1}\right)$, $\left(\mathrm{H}_{2}\right)$, and $\left(\mathrm{H}_{3}\right)$ hold, then one has

$$
\begin{gathered}
\left|\Pi_{h} u-\tilde{u}\right|_{1}+|u-\tilde{u}|_{1}+\left|u_{t}-\tilde{u}_{t}\right|_{1}+\left|u_{t t}-\tilde{u}_{t t}\right|_{1} \leq c h, \quad t \in[0, T] \\
\|u-\tilde{u}\|+\left\|u_{t}-\tilde{u}_{t}\right\|+\left\|u_{t t}-\tilde{u}_{t t}\right\| \leq c h^{2}, \quad t \in[0, T] .
\end{gathered}
$$

Next, we give the corresponding error estimates. Choosing $t=t_{n-1}, t_{n}, t_{n+1}$ in the first equation of (2.8), then multiplying the three equations by $1 / 4,1 / 2,1 / 4$, respectively, and adding them, we have

$$
\left(u_{t t}^{n, 1 / 4}, v_{h}\right)+a^{*}\left(u_{t}^{n, 1 / 4}, v_{h}\right)+b^{*}\left(f^{1 / 4}\left(u^{n}\right), v_{h}\right)=0, \quad \forall v_{h} \in V_{h}, n=1,2, \ldots, N-1 .
$$

Similarly, taking $t=t_{n-1}, t_{n}, t_{n+1}$ in the first equation of (3.8), then multiplying the three equations by $1 / 4,1 / 2,1 / 4$, respectively, and adding them, we obtain

$$
a^{*}\left(u_{t}^{n, 1 / 4}-\widetilde{u}_{t}^{n, 1 / 4}, v_{h}\right)+b^{*}\left(f^{1 / 4}\left(u^{n}\right)-f^{1 / 4}\left(\widetilde{u}^{n}\right), v_{h}\right)=0, \quad \forall v_{h} \in V_{h}, n=1,2, \ldots, N-1 .
$$

Subtracting (3.11) from (2.10) and using (3.12), we get the error equation

$$
\left(\partial_{t t}^{2} u_{h}^{n}-u_{t t}^{n, 1 / 4}, v_{h}\right)+a^{*}\left(\partial_{t} u_{h}^{n}-\tilde{u}_{t}^{n, 1 / 4}, v_{h}\right)+b^{*}\left(f^{(1 / 4) *}\left(u_{h}^{n}\right)-f^{1 / 4}\left(\tilde{u}^{n}\right), v_{h}\right)=0, \quad \forall v_{h} \in V_{h}
$$

where $n=1,2, \ldots, N-1$. Let

$$
u_{h}^{n}-u^{n}=\theta^{n}+\rho^{n}, \quad \theta^{n}=u_{h}^{n}-\tilde{u}^{n}, \quad \rho^{n}=\tilde{u}^{n}-u^{n}, \quad n=1,2, \ldots, N-1 .
$$

Then the error equation (3.13) can be rewritten as

$$
\begin{aligned}
\left(\partial_{t t}^{2} \theta^{n}, v_{h}\right)+a^{*}\left(\partial_{t} \theta^{n}, v_{h}\right)+b^{*}\left(f^{(1 / 4) *}\left(u_{h}^{n}\right)-f^{1 / 4}\left(\widetilde{u}^{n}\right), v_{h}\right) \\
=-\left(\partial_{t t}^{2} \rho^{n}, v_{h}\right)+\left(u_{t t}^{n, 1 / 4}-\partial_{t t}^{2} u_{h^{\prime}}^{n} v_{h}\right)+a^{*}\left(\rho_{t}^{n, 1 / 4}-\partial_{t} \rho^{n}, v_{h}\right) \\
+a^{*}\left(u_{t}^{n, 1 / 4}-\partial_{t} u^{n}, v_{h}\right), \quad \forall v_{h} \in V_{h}, n=1,2, \ldots, N-1
\end{aligned}
$$

Theorem 3.2. Let $\left\{u_{h}^{n}\right\}_{n=0}^{N}$ and $\tilde{u}$ be the solutions of (2.10) and (3.8), respectively. If the assumptions $\left(H_{1}\right),\left(H_{2}\right)$, and $\left(H_{3}\right)$ hold, then there exists a positive constant $h_{0}$, such that when $h<h_{0}$

$$
\begin{gathered}
\left|u_{h}^{1}-\tilde{u}^{1}\right|_{1}=\left|\theta^{1}\right|_{1} \leq c\left(h^{2}+\tau^{2}\right), \\
\left\|\partial_{t}\left(u_{h}^{0}-\tilde{u}^{0}\right)\right\|=\left|\partial_{t} \theta^{0}\right| \leq c\left(h^{2}+\tau\right) .
\end{gathered}
$$


Proof. Firstly, we estimate $\left|\theta^{1}\right|_{1}$. Applying the Taylor formula,

$$
\tilde{u}^{1}=\tilde{u}(\tau)=\tilde{u}(0)+\tau \tilde{u}_{t}(0)+\frac{1}{2} \tau^{2} \tilde{u}_{t t}(\xi)=\tilde{u}^{0}+\tau \tilde{u}_{t}^{0}+\frac{1}{2} \tau^{2} \tilde{u}_{t t}(\xi), \quad \xi \in(0, \tau),
$$

and noting the definitions of $u_{h}^{1}$ and $\tilde{u}^{0}$ in (2.10) and (3.8), respectively, we know

$$
u_{h}^{1}-\tilde{u}^{1}=\theta^{1}=\tau\left(\Pi_{h} u_{1}-\tilde{u}_{t}^{0}\right)-\frac{1}{2} \tau^{2} \tilde{u}_{t t}(\xi), \quad \xi \in(0, \tau),
$$

so

$$
\left|\theta^{1}\right|_{1}^{2}=\left|\tau\left(\Pi_{h} u_{1}-\tilde{u}_{t}^{0}\right)-\frac{1}{2} \tau^{2} \tilde{u}_{t t}(\xi)\right|_{1}^{2} \leq 4 \tau^{2}\left(\left|\Pi_{h} u_{1}-u_{1}\right|_{1}^{2}+\left|u_{1}-\tilde{u}_{t}^{0}\right|_{1}^{2}\right)+\frac{1}{2} \tau^{4}\left|\tilde{u}_{t t}(\xi)\right|_{1}^{2} .
$$

Notice that $u_{1}=u_{t}(0)=u_{t}^{0},(2.13)$ and (3.9), there exists a positive constant $h_{0}$ such that, when $h<h_{0}$ we have

$$
\left|\theta^{1}\right|_{1}^{2} \leq c \tau^{2} h^{2}+\frac{1}{2} \tau^{4}\left(\left|\tilde{u}_{t t}(\xi)\right|_{1}+c h\right)^{2} \leq c\left(\tau^{2} h^{2}+\tau^{4}\right) \leq c\left(h^{4}+\tau^{4}\right)
$$

This implies (3.16) holds. Secondly, noting $\theta^{0}=0$ and (3.19), we obtain

$$
\partial_{t} \theta^{0}=\frac{\theta^{1}-\theta^{0}}{\tau}=\Pi_{h} u_{1}-\tilde{u}_{t}^{0}-\frac{1}{2} \tau \tilde{u}_{t t}(\xi), \quad \xi \in(0, \tau),
$$

hence, by (2.16), (3.10), and $h<h_{0}$ we have

$$
\begin{aligned}
\left\|\partial_{t} \theta^{0}\right\|^{2} & =\left\|\left(\Pi_{h} u_{1}-u_{1}\right)+\left(u_{1}-\tilde{u}_{t}^{0}\right)-\frac{1}{2} \tau \tilde{u}_{t t}(\xi)\right\|^{2} \\
& \leq 4\left\|\Pi_{h} u_{1}-u_{1}\right\|^{2}+4\left\|u_{1}-\tilde{u}_{t}^{0}\right\|+\frac{1}{2} \tau^{2}\left\|\tilde{u}_{t t}(\xi)\right\|^{2} \\
& \leq c h^{4}+\frac{1}{2} \tau^{2}\left(\left\|u_{t t}(\xi)\right\|+c h^{2}\right)^{2} \leq c\left(h^{4}+\tau^{2}\right) .
\end{aligned}
$$

This completes the proof of (3.17). 
Theorem 3.3. Let $u,\left\{u_{h}^{n}\right\}_{n=0}^{N}$ and $\tilde{u}$ be the solutions of (1.1), (2.10), and (3.8), respectively. Assume that $\left(H_{1}\right),\left(H_{2}\right)$, and $\left(H_{3}\right)$ hold; then for any $n=1,2, \ldots, N-1$, when $\tau$ is sufficiently small and $h<h_{0}$, one has

$$
\begin{aligned}
b^{*}\left(f^{(1 / 4) *}\left(u_{h}^{n}\right)-f^{1 / 4}\left(\tilde{u}^{n}\right), \Pi_{h}^{*} \partial_{t} \theta^{n}\right) \\
\geq \frac{1}{4 \tau} \sum_{j=1}^{r-1} \frac{1}{h_{j}}\left[a_{j-1 / 2}^{n+1}\left(\theta_{j}^{n+1}-\theta_{j-1}^{n+1}\right)^{2}-a_{j-1 / 2}^{n}\left(\theta_{j}^{n}-\theta_{j-1}^{n}\right)^{2}\right]-\frac{3}{4}\left|\partial_{t} \theta^{n}\right|_{1}^{2} \\
\quad-c\left[\left(1+\left|\theta_{x}^{n}\right|_{0, \infty}^{2}\right)\left|\theta^{n+1}\right|_{1}^{2}+\left|\theta^{n}\right|_{1}^{2}+\left|\theta^{n-1}\right|_{1}^{2}\right]-c \tau^{2},
\end{aligned}
$$

where

$$
a_{j-1 / 2}^{k}=\int_{0}^{1} f^{\prime}\left(\tilde{u}_{x}^{k}\left(x_{j-1 / 2}\right)-s \theta_{x}^{k}\left(x_{j-1 / 2}\right)\right) d s, \quad k=n, n+1, j=1,2, \ldots, r-1 .
$$

Proof. Expanding the term $b^{*}\left(f^{(1 / 4) *}\left(u_{h}^{n}\right)-f^{1 / 4}\left(\tilde{u}^{n}\right), \Pi_{h}^{*} \partial_{t} \theta^{n}\right)$, we have

$$
\begin{aligned}
& b^{*}\left(f^{(1 / 4) *}\left(u_{h}^{n}\right)-f^{1 / 4}\left(\tilde{u}^{n}\right), \Pi_{h}^{*} \partial_{t} \theta^{n}\right) \\
&=b^{*}\left(f^{1 / 4}\left(u_{h}^{n}\right)-f^{1 / 4}\left(\tilde{u}^{n}\right), \Pi_{h}^{*} \partial_{t} \theta^{n}\right)+b^{*}\left(\frac{1}{4}\left[f\left(u_{h}^{n}\right)-f\left(u_{h}^{n+1}\right)\right], \Pi_{h}^{*} \partial_{t} \theta^{n}\right) \\
&=\frac{1}{2 \tau}\left[b^{*}\left(f^{1 / 2}\left(u_{h}^{n}\right)-f^{1 / 2}\left(\tilde{u}^{n}\right), \Pi_{h}^{*} \theta^{n+1}\right)-b^{*}\left(f^{1 / 2}\left(u_{h}^{n-1}\right)-f^{1 / 2}\left(\tilde{u}^{n-1}\right), \Pi_{h}^{*} \theta^{n}\right)\right] \\
&-\frac{1}{2 \tau}\left[b^{*}\left(f^{1 / 2}\left(u_{h}^{n}\right)-f^{1 / 2}\left(\tilde{u}^{n}\right), \Pi_{h}^{*} \theta^{n}\right)-b^{*}\left(f^{1 / 2}\left(u_{h}^{n-1}\right)-f^{1 / 2}\left(\tilde{u}^{n-1}\right), \Pi_{h}^{*} \theta^{n+1}\right)\right] \\
&+\frac{1}{4 \tau}\left[b^{*}\left(f\left(u_{h}^{n}\right)-f\left(u_{h}^{n+1}\right), \Pi_{h}^{*} \theta^{n+1}\right)-b^{*}\left(f\left(u_{h}^{n}\right)-f\left(u_{h}^{n+1}\right), \Pi_{h}^{*} \theta^{n}\right)\right] .
\end{aligned}
$$

For any $v_{h} \in V_{h}$, since $v_{h}=\sum_{i=1}^{r-1} v_{h i} \psi_{i}(x)$ and $v_{h 0}=v_{h r}=0$, then

$$
b^{*}\left(f(u), v_{h}\right)=\sum_{i=1}^{r-1} v_{h i}\left[f\left(u_{x}\left(x_{i-1 / 2}\right)\right)-f\left(u_{x}\left(x_{i+1 / 2}\right)\right)\right]=\sum_{i=1}^{r} f\left(u_{x}\left(x_{i-1 / 2}\right)\right)\left(v_{h i}-v_{h i-1}\right) .
$$

Let $\theta^{i}\left(x_{j}\right)=\theta_{j}^{i}$, thus $\Pi_{h}^{*} \theta^{i}=\sum_{j=1}^{r-1} \theta_{j}^{i} \psi_{j}(x) \in V_{h}$. Applying transformation of variable, we know

$$
\begin{aligned}
& b^{*}\left(f^{1 / 2}\left(u_{h}^{m}\right)-f^{1 / 2}\left(\tilde{u}^{m}\right), \Pi_{h}^{*} \theta^{i}\right) \\
& \quad=\sum_{j=1}^{r}\left(\theta_{j}^{i}-\theta_{j-1}^{i}\right)\left[f^{1 / 2}\left(u_{h x}^{m}\left(x_{j-1 / 2}\right)\right)-f^{1 / 2}\left(\tilde{u}_{x}^{m}\left(x_{j-1 / 2}\right)\right)\right]
\end{aligned}
$$




$$
\begin{gathered}
=\frac{1}{2} \sum_{j=1}^{r}\left(\theta_{j}^{i}-\theta_{j-1}^{i}\right)\left\{\left[f\left(u_{h x}^{m+1}\left(x_{j-1 / 2}\right)\right)-f\left(\tilde{u}_{x}^{m+1}\left(x_{j-1 / 2}\right)\right)\right]\right. \\
\left.+\left[f\left(u_{h x}^{m}\left(x_{j-1 / 2}\right)\right)-f\left(\tilde{u}_{x}^{m}\left(x_{j-1 / 2}\right)\right)\right]\right\} \\
=\frac{1}{2} \sum_{j=1}^{r}\left(\theta_{j}^{i}-\theta_{j-1}^{i}\right)\left[a_{j-1 / 2}^{m+1} \theta_{x}^{m+1}\left(x_{j-1 / 2}\right)+a_{j-1 / 2}^{m} \theta_{x}^{m}\left(x_{j-1 / 2}\right)\right] \\
=\frac{1}{2} \sum_{j=1}^{r} \frac{1}{h_{j}}\left[a_{j-1 / 2}^{m+1}\left(\theta_{j}^{i}-\theta_{j-1}^{i}\right)\left(\theta_{j}^{m+1}-\theta_{j-1}^{m+1}\right)\right. \\
\left.+a_{j-1 / 2}^{m}\left(\theta_{j}^{i}-\theta_{j-1}^{i}\right)\left(\theta_{j}^{m}-\theta_{j-1}^{m}\right)\right],
\end{gathered}
$$

where

$$
\begin{aligned}
& a_{j-1 / 2}^{k}=\int_{0}^{1} f^{\prime}\left(\tilde{u}_{x}^{k}\left(x_{j-1 / 2}\right)+s \theta_{x}^{k}\left(x_{j-1 / 2}\right)\right) d s, \\
& \theta_{x}^{k}\left(x_{j-1 / 2}\right)=\frac{1}{h_{j}}\left(\theta_{j}^{k}-\theta_{j-1}^{k}\right), \quad k=m, m+1 .
\end{aligned}
$$

By (3.28), we have

$$
\begin{aligned}
& b^{*}\left(f^{1 / 2}\left(u_{h}^{n}\right)-f^{1 / 2}\left(\tilde{u}^{n}\right), \Pi_{h}^{*} \theta^{n+1}\right)=\frac{1}{2} \sum_{j=1}^{r} \frac{1}{h_{j}}\left[a_{j-1 / 2}^{n+1}\left(\theta_{j}^{n+1}-\theta_{j-1}^{n+1}\right)^{2}\right. \\
& \left.+a_{j-1 / 2}^{n}\left(\theta_{j}^{n+1}-\theta_{j-1}^{n+1}\right)\left(\theta_{j}^{n}-\theta_{j-1}^{n}\right)\right], \\
& b^{*}\left(f^{1 / 2}\left(u_{h}^{n-1}\right)-f^{1 / 2}\left(\tilde{u}^{n-1}\right), \Pi_{h}^{*} \theta^{n}\right)=\frac{1}{2} \sum_{j=1}^{r} \frac{1}{h_{j}}\left[a_{j-1 / 2}^{n}\left(\theta_{j}^{n}-\theta_{j-1}^{n}\right)^{2}\right. \\
& \left.+a_{j-1 / 2}^{n-1}\left(\theta_{j}^{n}-\theta_{j-1}^{n}\right)\left(\theta_{j}^{n-1}-\theta_{j-1}^{n-1}\right)\right], \\
& b^{*}\left(f^{1 / 2}\left(u_{h}^{n}\right)-f^{1 / 2}\left(\tilde{u}^{n}\right), \Pi_{h}^{*} \theta^{n}\right)=\frac{1}{2} \sum_{j=1}^{r} \frac{1}{h_{j}}\left[a_{j-1 / 2}^{n+1}\left(\theta_{j}^{n+1}-\theta_{j-1}^{n+1}\right)\left(\theta_{j}^{n}-\theta_{j-1}^{n}\right)\right. \\
& \left.+a_{j-1 / 2}^{n}\left(\theta_{j}^{n}-\theta_{j-1}^{n}\right)^{2}\right], \\
& b^{*}\left(f^{1 / 2}\left(u_{h}^{n-1}\right)-f^{1 / 2}\left(\tilde{u}^{n-1}\right), \Pi_{h}^{*} \theta^{n+1}\right)=\frac{1}{2} \sum_{j=1}^{r} \frac{1}{h_{j}}\left[a_{j-1 / 2}^{n}\left(\theta_{j}^{n+1}-\theta_{j-1}^{n+1}\right)\left(\theta_{j}^{n}-\theta_{j-1}^{n}\right)\right. \\
& \left.+a_{j-1 / 2}^{n-1}\left(\theta_{j}^{n+1}-\theta_{j-1}^{n+1}\right)\left(\theta_{j}^{n-1}-\theta_{j-1}^{n-1}\right)\right] .
\end{aligned}
$$


Abstract and Applied Analysis

13

On the other hand

$$
\begin{aligned}
& b^{*}\left(f\left(u_{h}^{n}\right)-f\left(u_{h}^{n+1}\right), \Pi_{h}^{*} \theta^{n+1}\right)-b^{*}\left(f\left(u_{h}^{n}\right)-f\left(u_{h}^{n+1}\right), \Pi_{h}^{*} \theta^{n}\right) \\
& =\sum_{j=1}^{r}\left[f\left(u_{h x}^{n}\left(x_{j-1 / 2}\right)\right)-f\left(u_{h x}^{n+1}\left(x_{j-1 / 2}\right)\right)\right]\left[\left(\theta_{j}^{n+1}-\theta_{j-1}^{n+1}\right)-\left(\theta_{j}^{n}-\theta_{j-1}^{n}\right)\right] \\
& =\sum_{j=1}^{r} a_{j-(1 / 2)}^{* n}\left(u_{h x}^{n}\left(x_{j-1 / 2}\right)-u_{h x}^{n+1}\left(x_{j-1 / 2}\right)\right)\left(\theta_{j}^{n+1}-\theta_{j}^{n}-\left(\theta_{j-1}^{n+1}-\theta_{j-1}^{n}\right)\right) \\
& =-\tau^{2} \sum_{j=1}^{r} a_{j-1 / 2}^{* n} \partial_{t} u_{h x}^{n}\left(x_{j-1 / 2}\right)\left(\partial_{t} \theta_{j}^{n}-\partial_{t} \theta_{j-1}^{n}\right),
\end{aligned}
$$

where

$$
a_{j-1 / 2}^{* n}=\int_{0}^{1} f^{\prime}\left(u_{h x}^{n}\left(x_{j-1 / 2}\right)\right)+s\left(u_{h x}^{n}\left(x_{j-1 / 2}\right)-u_{h x}^{n+1}\left(x_{j-1 / 2}\right)\right) d s .
$$

Combining the above five equalities with (3.26), we can get

$$
\begin{aligned}
b^{*}\left(f^{(1 / 4) *}\left(u_{h}^{n}\right)-f^{1 / 4}\left(\tilde{u}^{n}\right), \Pi_{h}^{*} \partial_{t} \theta^{n}\right) \\
=\frac{1}{4 \tau} \sum_{j=1}^{r} \frac{1}{h_{j}}\left[a_{j-1 / 2}^{n+1}\left(\theta_{j}^{n+1}-\theta_{j-1}^{n+1}\right)^{2}-a_{j-(1 / 2)}^{n}\left(\theta_{j}^{n}-\theta_{j-1}^{n}\right)^{2}\right] \\
\quad+\frac{1}{4 \tau} \sum_{j=1}^{r} \frac{1}{h_{j}} a_{j-1 / 2}^{n}\left(\theta_{j}^{n}-\theta_{j-1}^{n}\right)\left[\theta_{j}^{n+1}-\theta_{j-1}^{n+1}-\left(\theta_{j}^{n}-\theta_{j-1}^{n}\right)\right] \\
\quad+\frac{1}{4 \tau} \sum_{j=1}^{r} \frac{1}{h_{j}} a_{j-1 / 2}^{n-1}\left(\theta_{j}^{n-1}-\theta_{j-1}^{n-1}\right)\left[\theta_{j}^{n+1}-\theta_{j-1}^{n+1}-\left(\theta_{j}^{n}-\theta_{j-1}^{n}\right)\right] \\
\quad-\frac{1}{4 \tau} \sum_{j=1}^{r} \frac{1}{h_{j}}\left(a_{j-1 / 2}^{n+1}-a_{j-1 / 2}^{n}\right)\left(\theta_{j}^{n+1}-\theta_{j-1}^{n+1}\right)\left(\theta_{j}^{n}-\theta_{j-1}^{n}\right) \\
\quad-\frac{\tau}{4} \sum_{j=1}^{r} a_{j-1 / 2}^{* n}\left(\partial_{t} \theta_{j}^{n}-\partial_{t} \theta_{j-1}^{n}\right) \partial_{t} u_{h x}^{n}\left(x_{j-1 / 2}\right) \\
=B_{1}+B_{2}+B_{3}+B_{4}+B_{5} .
\end{aligned}
$$

Using the $\varepsilon$-inequality, we find

$$
\begin{aligned}
-B_{2} & =\frac{1}{4 \tau} \sum_{j=1}^{r} \frac{1}{h_{j}} a_{j-1 / 2}^{n}\left(\theta_{j}^{n}-\theta_{j-1}^{n}\right)\left[\theta_{j}^{n}-\theta_{j-1}^{n}-\left(\theta_{j}^{n+1}-\theta_{j-1}^{n+1}\right)\right] \\
& =-\frac{1}{4} \sum_{j=1}^{r} \frac{1}{h_{j}} a_{j-1 / 2}^{n}\left(\theta_{j}^{n}-\theta_{j-1}^{n}\right)\left(\partial_{t} \theta_{j}^{n}-\partial_{t} \theta_{j-1}^{n}\right)
\end{aligned}
$$




$$
\begin{aligned}
& \leq c \sum_{j=1}^{r} \frac{\left(\theta_{j}^{n}-\theta_{j-1}^{n}\right)^{2}}{h_{j}}+\frac{1}{16} \sum_{j=1}^{r} \frac{\left(\partial_{t} \theta_{j}^{n}-\partial_{t} \theta_{j-1}^{n}\right)^{2}}{h_{j}}=c\left|\theta^{n}\right|_{1}^{2}+\frac{1}{16}\left|\partial_{t} \theta^{n}\right|_{1}^{2}, \\
-B_{3} & =\frac{1}{4 \tau} \sum_{j=1}^{r} \frac{1}{h_{j}} a_{j-1 / 2}^{n-1}\left(\theta_{j}^{n-1}-\theta_{j-1}^{n-1}\right)\left[\theta_{j}^{n}-\theta_{j-1}^{n}-\left(\theta_{j}^{n+1}-\theta_{j-1}^{n+1}\right)\right] \\
& =-\frac{1}{4} \sum_{j=1}^{r} \frac{1}{h_{j}} a_{j-1 / 2}^{n-1}\left(\theta_{j}^{n-1}-\theta_{j-1}^{n-1}\right)\left(\partial_{t} \theta_{j}^{n}-\partial_{t} \theta_{j-1}^{n}\right) \leq c\left|\theta^{n-1}\right|_{1}^{2}+\frac{1}{16}\left|\partial_{t} \theta^{n}\right|_{1}^{2}, \\
-B_{5} & =\frac{\tau}{4} \sum_{j=1}^{r} a_{j-1 / 2}^{* n}\left(\partial_{t} \theta_{j}^{n}-\partial_{t} \theta_{j-1}^{n}\right) \partial_{t} u_{h x}^{n}\left(x_{j-1 / 2}\right) \\
& \leq \frac{1}{16}\left|\partial_{t} \theta^{n}\right|_{1}^{2}+c \tau^{2} \sum_{j=1}^{r} h_{j}\left(\partial_{t} u_{h x}^{n}\left(x_{j-1 / 2}\right)\right)^{2}=\frac{1}{16}\left|\partial_{t} \theta^{n}\right|_{1}^{2}+I_{1} .
\end{aligned}
$$

Now we estimate $I_{1}$. Noting (2.2), we have

$$
u_{h x}^{n}\left(x_{j-1 / 2}\right)=\frac{u_{h j}^{n}-u_{h j-1}^{n}}{h_{j}}=\frac{1}{h_{j}} \int_{x_{j-1 / 2}}^{x_{j}} u_{h x}^{n}(x) d x .
$$

Furthermore,

$$
\left(\partial_{t} u_{h x}^{n}\left(x_{j-1 / 2}\right)\right)^{2}=\left(\frac{1}{h_{j}} \int_{x_{j-1 / 2}}^{x_{j}} \partial_{t} u_{h x}^{n}(x) d x\right)^{2} \leq \frac{1}{h_{j}} \int_{x_{j-1}}^{x_{j}}\left(\partial_{t} u_{h x}^{n}(x)\right)^{2} d x .
$$

So

$$
\begin{aligned}
I_{1} & \leq c \tau^{2} \sum_{j=1}^{r} \int_{x_{j-1}}^{x_{j}}\left(\partial_{t} u_{h x}^{n}(x)\right)^{2} d x=c \tau^{2}\left|\partial_{t} u_{h}^{n}\right|_{1}^{2} \\
& \leq c \tau^{2}\left|\partial_{t} \theta^{n}\right|_{1}^{2}+c \tau^{2}\left|\partial_{t} \tilde{u}^{n}\right|_{1}^{2} \leq c_{1} \tau^{2}\left|\partial_{t} \theta^{n}\right|_{1}^{2}+c \tau^{2}\left|\partial_{t} \tilde{u}^{n}\right|_{1}^{2} .
\end{aligned}
$$

When $\tau$ is sufficiently small, we can take $\tau$ suitable such that $c_{1} \tau^{2} \leq 1 / 16$, from the above equalities we have

$$
I_{1} \leq \frac{1}{16}\left|\partial_{t} \theta^{n}\right|_{1}^{2}+c \tau^{2}\left|\partial_{t} \tilde{u}^{n}\right|_{1}^{2}
$$

Moreover, in view of $\partial_{t} \tilde{u}^{n}=(1 / \tau) \int_{t_{n}}^{t_{n+1}} \tilde{u}_{t} d t$ and (3.9), we obtain

$$
\begin{aligned}
\left|\partial_{t} \tilde{u}^{n}\right|_{1}^{2} & =\int_{0}^{1}\left(\partial_{t} \tilde{u}_{x}^{n}(x)\right)^{2} d x=\frac{1}{\tau^{2}} \int_{0}^{1}\left(\int_{t_{n}}^{t_{n+1}} \tilde{u}_{t} d t\right)^{2} d x \leq \frac{1}{\tau} \int_{0}^{1} \int_{t_{n}}^{t_{n+1}}\left(\tilde{u}_{t}\right)^{2} d t d x \\
& =\frac{1}{\tau} \int_{t_{n}}^{t_{n+1}}\left|\tilde{u}_{t}\right|_{1}^{2} d t \leq \frac{1}{\tau} \int_{t_{n}}^{t_{n+1}}\left(c h+\left|u_{t}\right|_{1}\right)^{2} d t \leq c .
\end{aligned}
$$


Then

$$
I_{1} \leq \frac{1}{16}\left|\partial_{t} \theta^{n}\right|_{1}^{2}+c \tau^{2}
$$

Substituting the estimate of $I_{1}$ into (3.34), we have

$$
\begin{gathered}
-B_{5} \leq \frac{1}{8}\left|\partial_{t} \theta^{n}\right|_{1}^{2}+c \tau^{2}, \\
-B_{4}=\frac{1}{4 \tau} \sum_{j=1}^{r} \frac{1}{h_{j}}\left(a_{j-1 / 2}^{n+1}-a_{j-1 / 2}^{n}\right)\left(\theta_{j}^{n+1}-\theta_{j-1}^{n+1}\right)\left(\theta_{j}^{n}-\theta_{j-1}^{n}\right) .
\end{gathered}
$$

Since

$$
\begin{aligned}
a_{j-1 / 2}^{n+1}-a_{j-1 / 2}^{n}= & \int_{0}^{1}\left[f^{\prime}\left(\tilde{u}_{x}^{n+1}\left(x_{j-1 / 2}\right)+s \theta_{x}^{n+1}\left(x_{j-1 / 2}\right)\right)\right. \\
& \left.-f^{\prime}\left(\tilde{u}_{x}^{n}\left(x_{j-1 / 2}\right)+s \theta_{x}^{n}\left(x_{j-1 / 2}\right)\right)\right] d s \\
= & \int_{0}^{1} f^{\prime \prime}\left(\xi_{j}^{n}\right)\left[\tilde{u}_{x}^{n+1}\left(x_{j-1 / 2}\right)-\tilde{u}_{x}^{n}\left(x_{j-1 / 2}\right)\right. \\
& \left.+s\left(\theta_{x}^{n+1}\left(x_{j-1 / 2}\right)-\theta_{x}^{n}\left(x_{j-1 / 2}\right)\right)\right] d s \\
= & \tau \int_{0}^{1} f^{\prime \prime}\left(\xi_{j}^{n}\right) d s \partial_{t} \tilde{u}_{x}^{n}\left(x_{j-1 / 2}\right)+\tau \int_{0}^{1} f^{\prime \prime}\left(\xi_{j}^{n}\right) s d s \partial_{t} \theta_{x}^{n}\left(x_{j-1 / 2}\right)
\end{aligned}
$$

where $\xi_{j}^{n}$ lies between $\tilde{u}_{x}^{n+1}\left(x_{j-1 / 2}\right)+s \theta_{x}^{n+1}\left(x_{j-1 / 2}\right)$ and $\tilde{u}_{x}^{n}\left(x_{j-1 / 2}\right)+s \theta_{x}^{n}\left(x_{j-1 / 2}\right)$, by (2.13), (3.9), and the inverse estimate, we obtain

$$
\begin{aligned}
\left|\tilde{u}_{t}\right|_{1, \infty} & \leq\left|u_{t}\right|_{1, \infty}+\left|u_{t}-\tilde{u}_{t}\right|_{1, \infty} \leq\left|u_{t}\right|_{1, \infty}+\left|u_{t}-\Pi_{h} u_{t}\right|_{1, \infty}+\left|\Pi_{h} u_{t}-\tilde{u}_{t}\right|_{1, \infty} \\
& \leq\left|u_{t}\right|_{1, \infty}+c h\left|u_{t}\right|_{2, \infty}+c h^{-1 / 2}\left|\Pi_{h} u_{t}-\tilde{u}_{t}\right|_{1} \leq\left|u_{t}\right|_{1, \infty}+c h\left|u_{t}\right|_{2, \infty}+c h^{1 / 2}
\end{aligned}
$$

Thus, when $h<h_{0}$ we have $\left\|\tilde{u}_{x t}\right\|_{L^{\infty}\left(L^{\infty}\right)} \leq c$, then

$$
\left|\partial_{t} \tilde{u}_{x}^{n}\left(x_{j-1 / 2}\right)\right|=\left|\tilde{u}_{x t}\left(x_{j-1 / 2}, t_{n}+s \tau\right)\right| \leq\left\|\tilde{u}_{x t}\right\|_{L^{\infty}\left(L^{\infty}\right)} \leq c .
$$

From the assumption $\left(\mathrm{H}_{1}\right)$, we find that $\int_{0}^{1} f^{\prime \prime}\left(\xi_{j}^{n}\right) d s$ is bounded: this leads to the boundedness of $\int_{0}^{1} f^{\prime \prime}\left(\xi_{j}^{n}\right) d s \partial_{t} \tilde{u}_{x}^{n}\left(x_{j-1 / 2}\right)$ and $\int_{0}^{1} f^{\prime \prime}\left(\xi_{j}^{n}\right) s d s$. So

$$
\begin{aligned}
-B_{4}= & \frac{1}{4} \sum_{j=1}^{r} \frac{1}{h_{j}}\left[\int_{0}^{1} f^{\prime \prime}\left(\xi_{j}^{n}\right) d s \partial_{t} \tilde{u}_{x}^{n}\left(x_{j-1 / 2}\right)+\int_{0}^{1} f^{\prime \prime}\left(\xi_{j}^{n}\right) s d s \partial_{t} \theta_{x}^{n}\left(x_{j-1 / 2}\right)\right] \\
& \times\left(\theta_{j}^{n+1}-\theta_{j-1}^{n+1}\right)\left(\theta_{j}^{n}-\theta_{j-1}^{n}\right)
\end{aligned}
$$




$$
\begin{aligned}
= & \frac{1}{4} \sum_{j=1}^{r} \frac{1}{h_{j}}\left[\int_{0}^{1} f^{\prime \prime}\left(\xi_{j}^{n}\right) d s \partial_{t} \tilde{u}_{x}^{n}\left(x_{j-1 / 2}\right)\right]\left(\theta_{j}^{n+1}-\theta_{j-1}^{n+1}\right)\left(\theta_{j}^{n}-\theta_{j-1}^{n}\right) \\
& +\frac{1}{4} \sum_{j=1}^{r} \frac{1}{h_{j}}\left(\int_{0}^{1} f^{\prime \prime}\left(\xi_{j}^{n}\right) s d s\right)\left(\partial_{t} \theta_{j}^{n}-\partial_{t} \theta_{j-1}^{n}\right)\left(\theta_{j}^{n+1}-\theta_{j-1}^{n+1}\right) \theta_{x}^{n}\left(x_{j-1 / 2}\right) \\
\leq & c\left|\theta^{n+1}\right|_{1}^{2}+c\left|\theta^{n}\right|_{1}^{2}+c\left|\theta_{x}^{n}\right|_{0, \infty}\left|\theta^{n+1}\right|_{1}\left|\partial_{t} \theta^{n}\right|_{1} \\
\leq & c\left[\left(1+\left|\theta_{x}^{n}\right|_{0, \infty}\right)\left|\theta^{n+1}\right|_{1}^{2}+\left|\theta^{n}\right|_{1}^{2}\right]+\frac{1}{2}\left|\partial_{t} \theta^{n}\right|_{1}^{2} .
\end{aligned}
$$

Substituting the estimates of $B_{2}, B_{3}, B_{4}$, and $B_{5}$ into (3.33), we can derive (3.24). This completes the proof of the theorem.

Theorem 3.4. Let $u,\left\{u_{h}^{n}\right\}_{n=0}^{N}$ and $\tilde{u}$ be the solutions of (1.1), (2.10), and (3.8), respectively. Assume that $\left(H_{1}\right),\left(H_{2}\right)$, and $\left(H_{3}\right)$ hold; then for any $n=1,2, \ldots, N-1$, when $h$ and $\tau$ are sufficiently small, one has

$$
\max _{1 \leq n \leq N-1}\left\|\partial_{t} \theta^{n}\right\|+\left|\theta^{n}\right|_{1} \leq c\left(h^{2}+\tau\right)
$$

Proof. Taking $v_{h}=\Pi_{h}^{*} \partial_{t} \theta^{n}$ in the error equation (3.15) and making a simple calculation yield

$$
\begin{aligned}
\left(\partial_{t t}^{2} \theta^{n},\right. & \left.\Pi_{h}^{*} \partial_{t} \theta^{n}\right)+a^{*}\left(\partial_{t} \theta^{n}, \Pi_{h}^{*} \partial_{t} \theta^{n}\right)+b^{*}\left(f^{(1 / 4) *}\left(u_{h}^{n}\right)-f^{1 / 4}\left(\tilde{u}^{n}\right), \Pi_{h}^{*} \partial_{t} \theta^{n}\right) \\
= & -\left(\partial_{t t}^{2} \rho^{n}, \Pi_{h}^{*} \partial_{t} \theta^{n}\right)+\left(u_{t t}^{n, 1 / 4}-\partial_{t t}^{2} u^{n}, \Pi_{h}^{*} \partial_{t} \theta^{n}\right) \\
& +a^{*}\left(\rho_{t}^{n, 1 / 4}-\partial_{t} \rho^{n}, \Pi_{h}^{*} \partial_{t} \theta^{n}\right)+a^{*}\left(u_{t}^{n, 1 / 4}-\partial_{t} u^{n}, \Pi_{h}^{*} \partial_{t} \theta^{n}\right)
\end{aligned}
$$

Form (3.4), we derive that

$$
\begin{gathered}
\left(\partial_{t} \theta^{n}, \Pi_{h}^{*} \partial_{t} \theta^{n}\right)-2\left(\partial_{t} \theta^{n-1}, \Pi_{h}^{*} \partial_{t} \theta^{n}\right)+\left(\partial_{t} \theta^{n-1}, \Pi_{h}^{*} \partial_{t} \theta^{n-1}\right) \\
=n\left(\partial_{t} \theta^{n}-\partial_{t} \theta^{n-1}, \Pi_{h}^{*}\left(\partial_{t} \theta^{n}-\partial_{t} \theta^{n-1}\right)\right) \geq 0 .
\end{gathered}
$$


Then

$$
\begin{aligned}
\left(\partial_{t t}^{2} \theta^{n}, \Pi_{h}^{*} \partial_{t} \theta^{n}\right)= & \frac{1}{\tau}\left(\partial_{t} \theta^{n}-\partial_{t} \theta^{n-1}, \Pi_{h}^{*} \partial_{t} \theta^{n}\right) \\
= & \frac{1}{2 \tau}\left\{\left(\partial_{t} \theta^{n}, \Pi_{h}^{*} \partial_{t} \theta^{n}\right)-\left(\partial_{t} \theta^{n-1}, \Pi_{h}^{*} \partial_{t} \theta^{n-1}\right)\right. \\
& \left.\quad+\left[\left(\partial_{t} \theta^{n}, \Pi_{h}^{*} \partial_{t} \theta^{n}\right)-2\left(\partial_{t} \theta^{n-1}, \Pi_{h}^{*} \partial_{t} \theta^{n}\right)+\left(\partial_{t} \theta^{n-1}, \Pi_{h}^{*} \partial_{t} \theta^{n-1}\right)\right]\right\} \\
\geq & \frac{1}{2 \tau}\left\{\left|\left\|\partial_{t} \theta^{n}\right\|\right|_{0}^{2}-\left|\left\|\partial_{t} \theta^{n-1}\right\|\right|_{0}^{2}\right\} .
\end{aligned}
$$

By (3.7), we get

$$
a^{*}\left(\partial_{t} \theta^{n}, \Pi_{h}^{*} \partial_{t} \theta^{n}\right)=\left|\partial_{t} \theta^{n}\right|_{1}^{2}
$$

Applying Taylor's formula with integral-type remainder,

$$
f(t)=f\left(t_{n}\right)+f^{\prime}\left(t_{n}\right)\left(t-t_{n}\right)+\cdots+\frac{f^{(k)}\left(t_{n}\right)}{k !}\left(t-t_{n}\right)^{k}+\frac{1}{k !} \int_{t_{n}}^{t} f^{(k+1)}(s)(t-s)^{k} d s,
$$

we have

$$
\begin{gathered}
\partial_{t t}^{2} \rho^{n}=\frac{1}{\tau^{2}}\left[\int_{t_{n}}^{t_{n+1}}\left(t_{n+1}-s\right) \rho_{t t}(s) d s+\int_{t_{n}}^{t_{n-1}}\left(t_{n-1}-s\right) \rho_{t t}(s) d s\right] \\
=\frac{1}{\tau^{2}} \int_{-\tau}^{\tau}(\tau-|s|) \rho_{t t}\left(t_{n}+s\right) d s=\frac{1}{\tau^{2}} \int_{t_{n-1}}^{t_{n+1}}\left(\tau-\left|t_{n}-s\right|\right) \rho_{t t}(s) d s, \\
u_{t t}^{n, 1 / 4}-\partial_{t t}^{2} u^{n}=\int_{-\tau}^{\tau}(\tau-|s|)\left[\frac{1}{4}-\frac{(\tau-|s|)^{2}}{6 \tau^{2}}\right] u_{t t t t}\left(t_{n}+s\right) d s \\
=\int_{t_{n-1}}^{t_{n+1}}\left(\tau-\left|t_{n}-s\right|\right)\left[\frac{1}{4}-\frac{\left(\tau-\left|t_{n}-s\right|\right)^{2}}{6 \tau^{2}}\right] u_{t t t t}(s) d s, \\
u_{t}^{n, 1 / 4}-\partial_{t} u^{n}=\frac{1}{4}\left[\int_{t_{n}}^{t_{n+1}} u_{t t}(s) d s+\int_{t_{n}}^{t_{n-1}} u_{t t}(s) d s\right]-\int_{t_{n}}^{t_{n+1}} u_{t t}(s) \frac{t_{n+1}-s}{\tau} d s, \\
\rho_{t}^{n, 1 / 4}-\partial_{t} \rho^{n}=\frac{1}{4}\left[\int_{t_{n}}^{t_{n+1}} \rho_{t t}(s) d s+\int_{t_{n}}^{t_{n-1}} \rho_{t t}(s) d s\right]-\int_{t_{n}}^{t_{n+1}} \rho_{t t}(s) \frac{t_{n+1}-s}{\tau} d s .
\end{gathered}
$$

Note that $\left\|\Pi_{h}^{*} \partial_{t} \theta^{n}\right\| \leq c\left\|\partial_{t} \theta^{n}\right\|$, we get

$$
\begin{aligned}
\left(\partial_{t t}^{2} \rho^{n}, \Pi_{h}^{*} \partial_{t} \theta^{n}\right) & \leq\left\|\partial_{t t}^{2} \rho^{n}\right\|\left\|\Pi_{h}^{*} \partial_{t} \theta^{n}\right\| \leq c\left\|\partial_{t t}^{2} \rho^{n}\right\|^{2}+c\left\|\partial_{t} \theta^{n}\right\|^{2} \\
& \leq c \tau^{-1} \int_{t_{n-1}}^{t_{n+1}}\left\|\rho_{t t}\right\|^{2} d t+c\left\|\partial_{t} \theta^{n}\right\|^{2}
\end{aligned}
$$




$$
\begin{aligned}
\left(u_{t t}^{n, 1 / 4}-\partial_{t t}^{2} u^{n}, \Pi_{h}^{*} \partial_{t} \theta^{n}\right) & \leq\left\|u_{t t}^{n, 1 / 4}-\partial_{t t}^{2} u^{n}\right\|\left\|\Pi_{h}^{*} \partial_{t} \theta^{n}\right\| \leq c\left\|u_{t t}^{n, 1 / 4}-\partial_{t t}^{2} u^{n}\right\|^{2}+\left\|\partial_{t} \theta^{n}\right\|^{2} \\
& \leq c \tau^{3} \int_{t_{n-1}}^{t_{n+1}}\left\|u_{t t t t}\right\|^{2} d t+c\left\|\partial_{t} \theta^{n}\right\|^{2}
\end{aligned}
$$

For $\Pi_{h}^{*} v=\sum_{j=1}^{r-1} v_{j} \psi_{j}(x) \in V_{h}, v_{0}=v_{r}=0$, we have

$$
\begin{aligned}
a^{*}\left(u, \Pi_{h}^{*} v\right) & =\sum_{j=1}^{r-1} v_{j}\left(u_{x}\left(x_{j-1 / 2}\right)-u_{x}\left(x_{j+1 / 2}\right)\right)=\sum_{j=1}^{r} u_{x}\left(x_{j-1 / 2}\right)\left(v_{j}-v_{j-1}\right) \\
& \leq\left(\sum_{j=1}^{r} \frac{\left(v_{j}-v_{j-1}\right)^{2}}{h_{j}}\right)^{1 / 2}\left(\sum_{j=1}^{r} h_{j} u_{x}^{2}\left(x_{j-1 / 2}\right)\right)^{1 / 2} .
\end{aligned}
$$

Noting that

$$
\begin{aligned}
u_{x}\left(x_{j-1 / 2}\right)=u_{x}(x)-\int_{x_{j-1 / 2}}^{x} u_{x x}(s) d s, \quad x \in\left[x_{j-1}, x_{j}\right] \\
u_{x}^{2}\left(x_{j-1 / 2}\right)=\left(u_{x}(x)-\int_{x_{j-1 / 2}}^{x} u_{x x}(s) d s\right)^{2} \leq 2 u_{x}^{2}(x)+2\left(\int_{x_{j-1} / 2}^{x} u_{x x}(s) d s\right)^{2} \\
\leq 2 u_{x}^{2}(x)+2\left(\int_{x_{j-1}}^{x_{j}}\left|u_{x x}(x)\right| d x\right)^{2} \leq 2 u_{x}^{2}(x)+2 h_{j}\left(\int_{x_{j-1}}^{x_{j}} u_{x x}^{2}(x) d x\right),
\end{aligned}
$$

we see that

$$
\begin{aligned}
\sum_{j=1}^{r} h_{j} u_{x}^{2}\left(x_{j-1 / 2}\right)= & \sum_{j=1}^{r} \int_{x_{j-1}}^{x_{j}} u_{x}^{2}\left(x_{j-1 / 2}\right) d x \leq 2 \sum_{j=1}^{r} \int_{x_{j-1}}^{x_{j}} u_{x}^{2}(x) d x \\
& +2 \sum_{j=1}^{r} h_{j}^{2} \int_{x_{j-1}}^{x_{j}} u_{x x}^{2}(x) d x \leq 2|u|_{1}^{2}+2 h^{2}|u|_{2}^{2}
\end{aligned}
$$

So

$$
\begin{aligned}
a^{*}\left(u_{t}^{n, 1 / 4}-\partial_{t} u^{n}, \Pi_{h}^{*} \partial_{t} \theta^{n}\right) & \leq 2\left(\left|u_{t}^{n, 1 / 4}-\partial_{t} u^{n}\right|_{1}^{2}+h^{2}\left|u_{t}^{n, 1 / 4}-\partial_{t} u^{n}\right|_{2}^{2}\right)^{1 / 2}\left|\partial_{t} \theta^{n}\right|_{1} \\
& \leq c\left(\left|u_{t}^{n, 1 / 4}-\partial_{t} u^{n}\right|_{1}^{2}+h^{2}\left|u_{t}^{n, 1 / 4}-\partial_{t} u^{n}\right|_{2}^{2}\right)+\frac{1}{8}\left|\partial_{t} \theta^{n}\right|_{1}^{2} \\
& \leq c \tau \int_{t_{n-1}}^{t_{n+1}}\left(\left|u_{t t}\right|_{1}^{2}+h^{2}\left|u_{t t}\right|_{2}^{2}\right) d t+\frac{1}{8}\left|\partial_{t} \theta^{n}\right|_{1}^{2}
\end{aligned}
$$


Abstract and Applied Analysis

$$
\begin{aligned}
a^{*}\left(\rho_{t}^{n, 1 / 4}-\partial_{t} \rho^{n}, \Pi_{h}^{*} \partial_{t} \theta^{n}\right) & \leq c \tau \int_{t_{n-1}}^{t_{n+1}}\left(\left|\rho_{t t}\right|_{1}^{2}+h^{2}\left|\rho_{t t}\right|_{2}^{2}\right) d t+\frac{1}{8}\left|\partial_{t} \theta^{n}\right|_{1}^{2} \\
& =c \tau \int_{t_{n-1}}^{t_{n+1}}\left(\left|\rho_{t t}\right|_{1}^{2}+h^{2}\left|u_{t t}\right|_{2}^{2}\right) d t+\frac{1}{8}\left|\partial_{t} \theta^{n}\right|_{1}^{2} .
\end{aligned}
$$

Substituting (3.24), (3.49)-(3.57) into (3.47) and using the Lemma 3.1, we obtain

$$
\begin{aligned}
\frac{1}{2 \tau}\left\{\left|\left\|\partial_{t} \theta^{n}\right\|\right|_{0}^{2}-\left|\left\|\partial_{t} \theta^{n-1}\right\|\right|_{0}^{2}\right\}+\frac{1}{4 \tau} \sum_{j=1}^{r} \frac{1}{h_{j}}\left[a_{j-1 / 2}^{n+1}\left(\theta_{j}^{n+1}-\theta_{j-1}^{n+1}\right)^{2}-a_{j-1 / 2}^{n}\left(\theta_{j}^{n}-\theta_{j-1}^{n}\right)^{2}\right] \\
\leq c \\
\qquad \partial_{t} \theta^{n} \|^{2}+c\left[\left(1+\left|\theta_{x}^{n}\right|_{0, \infty}^{2}\right)\left|\theta^{n+1}\right|_{1}^{2}+\left|\theta^{n}\right|_{1}^{2}+\left|\theta^{n-1}\right|_{1}^{2}\right] \\
+c\left(\tau^{2}+\tau^{4}+\tau^{2} h^{2}+h^{4}\right) .
\end{aligned}
$$

Multiplying the above inequation by $\tau$ and then summing it with respect to $n$ from 1 to $n$, we have

$$
\begin{aligned}
\frac{1}{2}\left|\left\|\partial_{t} \theta^{n}\right\|\right|_{0}^{2}+\frac{1}{4} \sum_{j=1}^{r} \frac{1}{h_{j}} a_{j-1 / 2}^{n+1}\left(\theta_{j}^{n+1}-\theta_{j-1}^{n+1}\right)^{2} \\
\leq \frac{1}{2}\left|\left\|\partial_{t} \theta^{0}\right\|\right|_{0}^{2}+\frac{1}{4} \sum_{j=1}^{r} \frac{1}{h_{j}} a_{j-1 / 2}^{n+1}\left(\theta_{j}^{1}-\theta_{j-1}^{1}\right)^{2} \\
\quad+c \tau \sum_{i=1}^{n}\left[\left\|\partial_{t} \theta^{i}\right\|^{2}+\left(1+\left|\theta_{x}^{n}\right|_{0, \infty}^{2}\right)\left|\theta^{i+1}\right|_{1}^{2}+\left|\theta^{i}\right|_{1}^{2}+\left|\theta^{i-1}\right|_{1}^{2}\right]+c\left(\tau^{2}+h^{4}\right) .
\end{aligned}
$$

Now we make the induction hypothesis:

$$
\max _{1 \leq k \leq n}\left|\theta_{x}^{k}\right|_{0, \infty} \leq 1
$$

Substituting (3.60) into (3.59) and noting (3.6) and the assumption $\left(\mathrm{H}_{1}\right)$, we conclude

$$
\begin{aligned}
c_{2}\left(\left\|\partial_{t} \theta^{n}\right\|^{2}+\left|\theta^{n+1}\right|_{1}^{2}\right) & \leq \frac{1}{2} \sqrt{\frac{3}{4}}\left\|\partial_{t} \theta^{n}\right\|^{2}+\frac{1}{4} l\left|\theta^{n+1}\right|_{1}^{2} \\
& \leq\left.\frac{1}{2}\left\|\partial_{t} \theta^{n}\right\|\right|_{0} ^{2}+\frac{1}{4} \sum_{j=1}^{r} \frac{1}{h_{j}} a_{j-1 / 2}^{n+1}\left(\theta_{j}^{n+1}-\theta_{j-1}^{n+1}\right)^{2}
\end{aligned}
$$




$$
\begin{aligned}
\leq & \frac{1}{2} \sqrt{\frac{3}{2}}\left\|\partial_{t} \theta^{0}\right\|^{2}+\frac{1}{4} l\left|\theta^{1}\right|_{1}^{2} \\
& +c \tau \sum_{i=1}^{n}\left\|\partial_{t} \theta^{i}\right\|^{2}+c \tau \sum_{i=0}^{n+1}\left|\theta^{i}\right|_{1}^{2}+c\left(\tau^{2}+h^{4}\right) \\
\leq & c_{3}\left(\left\|\partial_{t} \theta^{0}\right\|^{2}+\left|\theta^{1}\right|_{1}^{2}+\tau^{2}+h^{4}+\tau \sum_{i=1}^{n}\left\|\partial_{t} \theta^{i}\right\|^{2}+\tau \sum_{i=0}^{n+1}\left|\theta^{i}\right|_{1}^{2}\right) .
\end{aligned}
$$

Taking $\tau$ is sufficiently small, such that $c_{3} \tau<c_{2}$ and $c_{1} \tau^{2} \leq 1 / 16$ (these ensure that Theorem 3.3. holds), combining the above equalities and Theorem 3.2. we have

$$
\left\|\partial_{t} \theta^{n}\right\|^{2}+\left|\theta^{n+1}\right|_{1}^{2} \leq c\left(\tau^{2}+h^{4}\right)+\frac{c_{3} \tau}{c_{2}}\left(\sum_{i=1}^{n}\left\|\partial_{t} \theta^{i}\right\|^{2}+\sum_{i=0}^{n+1}\left|\theta^{i}\right|_{1}^{2}\right)
$$

By the discrete Gronwall's lemma, it follows that

$$
\left\|\partial_{t} \theta^{n}\right\|^{2}+\left|\theta^{n+1}\right|_{1}^{2} \leq c\left(\tau^{2}+h^{4}\right), \quad n=1,2, \ldots, N-1
$$

To complete our proof we must verify that (3.60) holds. In fact, by considering the inverse hypothesis $\left|\theta^{n}\right|_{1, \infty} \leq c_{4} h^{-1 / 2}\left|\theta^{n}\right|_{1}, n=1,2, \ldots, N-1$ and (3.16), we have

$$
\left|\theta_{x}^{1}\right|_{0, \infty}=\left|\theta^{1}\right|_{1, \infty} \leq c_{4} h^{-1 / 2} c\left(\tau^{2}+h^{2}\right) \leq c_{5} h^{-1 / 2}\left(\tau^{2}+h^{2}\right)
$$

Thus, we can choose $h, \tau$ such that $h, \tau$ satisfy $c_{5} h^{-1 / 2}\left(\tau^{2}+h^{2}\right) \leq 1$ for $h, \tau$ are sufficiently small, that is, (3.60) holds for $n=1$. From the proof of (3.63), as $h, \tau$ are sufficiently small and satisfy

$$
c_{1} \tau^{2} \leq \frac{1}{16}, \quad c_{3} \tau<c_{2}, \quad c_{5} h^{-1 / 2}\left(\tau^{2}+h^{2}\right) \leq 1,
$$

then (3.63) holds for $n=1$. Thus

$$
\left|\theta^{2}\right|_{1} \leq c_{6}\left(h^{2}+\tau\right) \text {. }
$$

Hence

$$
\left|\theta_{x}^{2}\right|_{0, \infty}=\left|\theta^{2}\right|_{1, \infty} \leq c_{4} h^{-1 / 2}\left|\theta^{2}\right|_{1} \leq c_{4} c_{6} h^{-1 / 2}\left(h^{2}+\tau\right) .
$$


Table 1: $\left\|u-u_{h}\right\|_{0, h}$

\begin{tabular}{llllllllll}
\hline$h$ & $t=0.1$ & $t=0.2$ & $t=0.3$ & $t=0.4$ & $t=0.5$ & $t=0.6$ & $t=0.7$ & $t=0.8$ & $t=0.9$ \\
\hline $1 / 20$ & 0.1025 & 0.0922 & 0.0831 & 0.0742 & 0.0671 & 0.0600 & 0.0548 & 0.0490 & 0.0447 \\
$1 / 40$ & 0.0520 & 0.0469 & 0.0424 & 0.0374 & 0.0346 & 0.0309 & 0.0279 & 0.0253 & 0.0229 \\
$1 / 80$ & 0.0261 & 0.0235 & 0.0212 & 0.0191 & 0.0173 & 0.0156 & 0.0141 & 0.0128 & 0.0116 \\
$1 / 160$ & 0.0131 & 0.0116 & 0.0106 & 0.0096 & 0.0087 & 0.0078 & 0.0071 & 0.0064 & 0.0058 \\
\hline
\end{tabular}

Table 2: $\left|u-u_{h}\right|_{1, h}$.

\begin{tabular}{lccccccccc}
\hline$h$ & $t=0.1$ & $t=0.2$ & $t=0.3$ & $t=0.4$ & $t=0.5$ & $t=0.6$ & $t=0.7$ & $t=0.8$ & $t=0.9$ \\
\hline $1 / 20$ & 0.3510 & 0.3165 & 0.2852 & 0.2569 & 0.2315 & 0.2088 & 0.1884 & 0.1702 & 0.1539 \\
$1 / 40$ & 0.1736 & 0.1567 & 0.1413 & 0.1274 & 0.1150 & 0.1038 & 0.0938 & 0.0849 & 0.0768 \\
$1 / 80$ & 0.0863 & 0.0779 & 0.0703 & 0.0634 & 0.0573 & 0.0518 & 0.0468 & 0.0424 & 0.0384 \\
$1 / 160$ & 0.0430 & 0.0388 & 0.0351 & 0.0316 & 0.0286 & 0.0258 & 0.0234 & 0.0212 & 0.0192 \\
\hline
\end{tabular}

Similarly, letting $h, \tau$ be sufficiently small such that $h, \tau$ satisfy $c_{4} c_{6} h^{-1 / 2}\left(h^{2}+\tau\right) \leq 1$, then we get $\left|\theta_{x}^{2}\right|_{0, \infty} \leq 1$. Furthermore, setting $h, \tau$ to satisfy

$$
c_{1} \tau^{2} \leq \frac{1}{16}, \quad c_{3} \tau<c_{2}, \quad c_{5} h^{-1 / 2}\left(\tau^{2}+h^{2}\right) \leq 1, \quad c_{4} c_{6} h^{-1 / 2}\left(h^{2}+\tau\right) \leq 1,
$$

we conclude that (3.60) holds for $n=2$. Using the above facts, we find that (3.63) holds for $n=2$ as $h, \tau$ are sufficiently small and satisfy (3.68). By the induction argument for $n=1,2, \ldots, N-1$, we deduce that (3.60) holds as $h, \tau$ satisfy (3.68). Then (3.63) holds for $n=1,2, \ldots, N-1$, which implies (3.46) is valid. This completes the proof of the theorem.

Note that $\theta^{0}=0$ and (2.13), by Theorems 3.2 and 3.4, Lemma 3.1, and the triangle inequality, we obtain the following.

Theorem 3.5. Under the assumption of Theorem 3.4, for $h, \tau$ are sufficiently small, one has

$$
\begin{gathered}
\max _{0 \leq n \leq N}\left\|u_{h}^{n}-u^{n}\right\| \leq c\left(h^{2}+\tau\right), \\
\max _{0 \leq n \leq N}\left|u_{h}^{n}-u^{n}\right|_{1} \leq c(h+\tau), \\
\max _{0 \leq n \leq N}\left|u_{h}^{n}-\tilde{u}^{n}\right|_{1} \leq c\left(h^{2}+\tau\right) .
\end{gathered}
$$

Remark 3.6. The optimal order error estimates in $L^{2}, H^{1}$ norms and superconvergence error estimates in $H^{1}$ norm of the solution of the finite volume scheme (2.10) are achieved in Theorem 3.5 . 
Table 3: The orders of $\left\|u-u_{h}\right\|_{0, h}$ and $\left|u-u_{h}\right|_{1, h}$.

\begin{tabular}{|c|c|c|c|c|c|c|}
\hline Time & Order & For & $\left\|u-u_{h}\right\|_{0, h}$ & Order & For & $\left|u-u_{h}\right|_{1, h}$ \\
\hline$t$ & $0.05 / 0.025$ & $0.025 / 0.0125$ & $0.0125 / 0.00625$ & $0.05 / 0.025$ & $0.025 / 0.0125$ & $0.0125 / 0.00625$ \\
\hline 0.1 & 0.9790 & 0.9947 & 1.0000 & 1.0157 & 1.0083 & 1.0050 \\
\hline 0.2 & 0.9752 & 0.9969 & 1.0185 & 1.0142 & 1.0083 & 1.0056 \\
\hline 0.3 & 0.9708 & 1.0000 & 1.0000 & 1.0132 & 1.0072 & 1.0021 \\
\hline 0.4 & 0.9884 & 0.9695 & 0.9925 & 1.0118 & 1.0068 & 1.0046 \\
\hline 0.5 & 0.9555 & 1.0000 & 0.9917 & 1.0094 & 1.0050 & 1.0025 \\
\hline 0.6 & 0.9574 & 0.9861 & 1.0000 & 1.0083 & 1.0028 & 1.0056 \\
\hline 0.7 & 0.9739 & 0.9846 & 0.9898 & 1.0061 & 1.0031 & 1.0000 \\
\hline 0.8 & 0.9536 & 0.9830 & 1.0000 & 1.0034 & 1.0017 & 1.0000 \\
\hline 0.9 & 0.9649 & 0.9812 & 1.0000 & 1.0028 & 1.0000 & 1.0000 \\
\hline
\end{tabular}

\section{Numerical Experiments}

To test the finite volume scheme (2.10) and the theoretical analysis, we do some experiments by considering the following problem:

$$
\begin{aligned}
& \text { (a) } u_{t t}=u_{x x t}+\frac{\pi^{2}-1}{\pi^{2}} u_{x x}, \quad(x, t) \in(0,1) \times(0,1], \\
& \text { (b) } u(x, 0)=\sin (\pi x), \quad u_{t}(x, 0)=-\sin (\pi x), \quad x \in(0,1), \\
& \text { (c) } u(0, t)=u(1, t)=0, \quad t \in[0,1] .
\end{aligned}
$$

The exact solution of the problem (4.1) is $u=e^{-t} \sin (\pi x),(x, t) \in[0,1] \times[0,1]$.

For simplicity, we choose a constant spatial step $h$ and a constant time step $\tau$. The corresponding nodes are denoted by $x_{i}=i h, i=0,1, \ldots, 2 r$ and the time levels by $t_{j}=j \tau$, $j=0,1, \ldots, 2 N$.

The discrete forms $\|u\|_{0, h}$ and $|u|_{1, h}$ of forms $\|u\|$ and $|u|_{1}$ are calculated by the compound Simpson formula. Here we choose $r=N$; this yields $h=\tau$. The program is written in MATLAB and run in Windows XP. By the calculated approximate solution $u_{h}$, we get the discrete norms $\left\|u-u_{h}\right\|_{0, h}$ and $\left|u-u_{h}\right|_{1, h}$, which are given in Tables 1 and 2 .

From Tables 1 and 2, we can see that the scheme (2.10) is indeed efficient. For $h=\tau$, we get the first-order optimal convergence under the discrete norm $\|u\|_{0, h}$ and the discrete norm $|u|_{1, h}$, respectively (see Table 3 ). Meanwhile, the scheme (2.10) is stable.

\section{Acknowledgments}

This project is supported by the fund of National Natural Science (11171193 and 10971254) and the fund of Natural Science of Shandong Province (ZR2011AM016 and ZR2009AZ003).

\section{References}

[1] G. Andrews, "On the existence of solutions to the equation $u_{t t}=u_{x x t}+\sigma\left(u_{x}\right)_{x}$ " Journal of Differential Equations, vol. 35, no. 2, pp. 200-231, 1980. 
[2] J. M. Greenberg, R. C. MacCamy, and V. J. Mizei, “On the existence, uniqueness and stability of solutions of the equation $\sigma^{\prime}\left(u_{x}\right) u_{x x}+\lambda u_{x x t}=\rho_{0} u_{t t}$," Journal of Mathematics and Mechanics, vol. 17, pp. 707-728, 1968.

[3] J. M. Greenberg, "On the existence, uniqueness, and stability of solutions of the equation $\rho_{0} X_{x x}=$ $E\left(X_{x}\right) X_{x x}+\lambda X_{x x t}$," Journal of Mathematical Analysis and Applications, vol. 25, pp. 575-591, 1969.

[4] J. M. Greenberg and R. C. MacCamy, "On the exponential stability of solutions of $E\left(u_{x}\right) u_{x x}+\lambda u_{x t x}=$ $\rho u_{t t}, "$ Journal of Mathematical Analysis and Applications, vol. 31, pp. 406-417, 1970.

[5] Y. Liu and D. Liu, "Initial-boundary problem, periodic boundary problem and initial value problem of equation $u_{t t}=u_{x x t}+\sigma\left(u_{x}\right)_{x}$," Chinese Annals of Mathematics, vol. 9, no. 4, pp. 459-470, 1988.

[6] X. B. Gao, G. H. Wan, and K. Z. Chen, "The finite difference method for the initial-boundary-value problem of the equation $u_{t t}=u_{x x t}+f\left(u_{x}\right)_{x}$, Mathematica Numerica Sinica, vol. 22, no. 2, pp. 167-178, 2000.

[7] Z. Jiang, S. Wang, G. Gao, and C. Chen, "Semi-discrete finite element methods for purely longitudinal motion of a homogeneous bar," SCI-TECH Engineering, vol. 7, pp. 1541-1545, 2007.

[8] Z. Jiang, C. Chen, S. Wang, and G. Gao, "Fully discrete scheme and error estimate for the purely longitudinal motion of a homogeneous bar," SCI-TECH Engineering, vol. 7, pp. 1836-1839, 2007.

[9] Z. Jiang and Y. Jiang, "Mixed finite element simulation for the initial value problems of purely longitudinal motion of a homogeneous bar," Journal of Jishou University, vol. 28, pp. 7-13, 2007.

[10] Z. Jiang and A. Li, "Expanded mixed finite element methods for the problem of purely longitudinal motion of a homogeneous bar," Journal of Computational and Applied Mathematics, vol. 235, no. 8, pp. 2157-2169, 2011.

[11] Z. Jiang and A. Zhu, "Finite volume method for an initial-boundary value problem for the purely longitudinal motion equation of a homogeneous bar," Journal of Systems Science and Mathematical Sciences, vol. 28, no. 7, pp. 867-885, 2008.

[12] M. Yang and Y. Yuan, "A multistep finite volume element scheme along characteristics for nonlinear convection diffusion problems," Mathematica Numerica Sinica, vol. 26, no. 4, pp. 484-496, 2004.

[13] M. Yang and Y. Yuan, "A symmetric characteristic FVE method with second order accuracy for nonlinear convection diffusion problems," Journal of Computational and Applied Mathematics, vol. 200, no. 2, pp. 677-700, 2007.

[14] C. Bi, "Mortar upwind finite volume element method for convection diffusion problems," Applied Mathematics and Computation, vol. 183, no. 2, pp. 831-841, 2006.

[15] S. Boivin, F. Cayre, and J. M. Herard, "A finite volume method to solve the Navier-Stokes equations for incompressible flows on unstructured meshes," International Journal of Thermal Sciences, vol. 39, no. 8, pp. 806-825, 2000. 


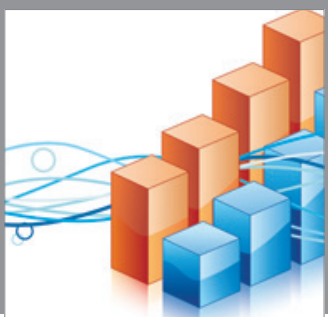

Advances in

Operations Research

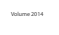

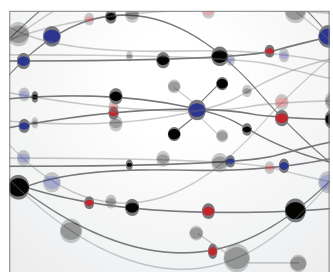

\section{The Scientific} World Journal
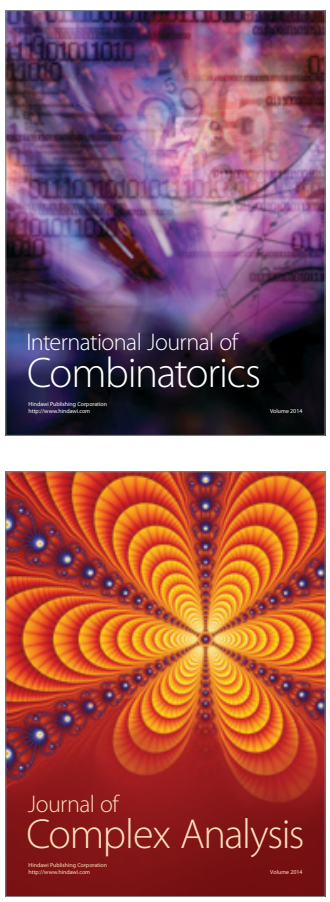

International Journal of

Mathematics and

Mathematical

Sciences
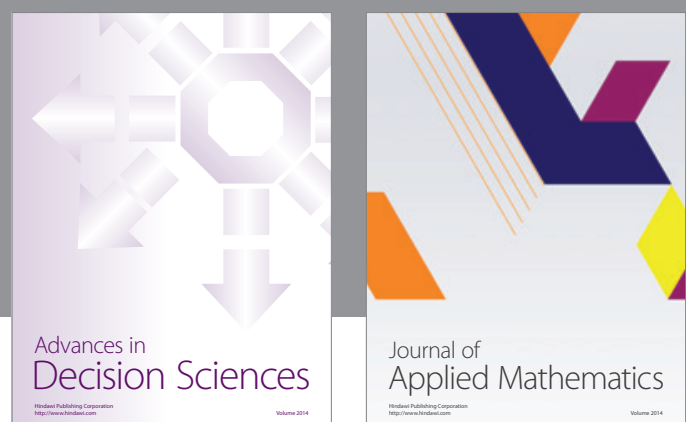

Journal of

Applied Mathematics
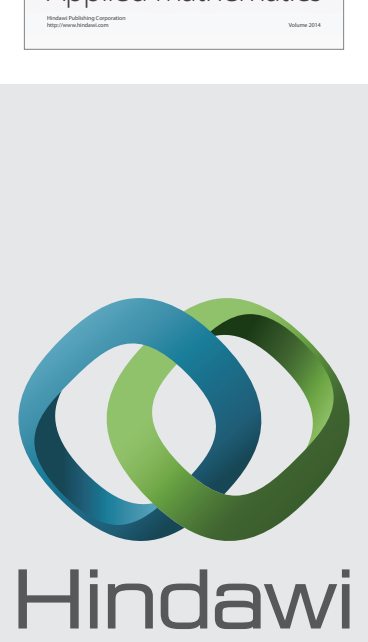

Submit your manuscripts at http://www.hindawi.com
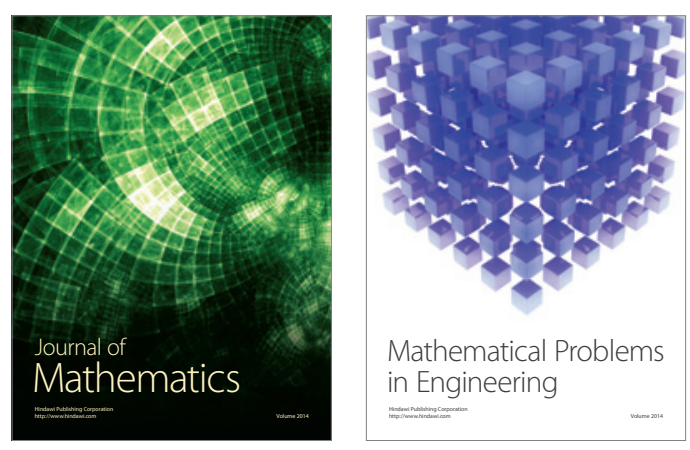

Mathematical Problems in Engineering
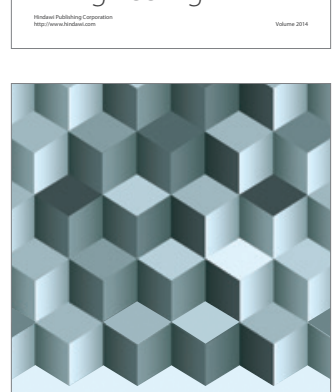

Journal of

Function Spaces
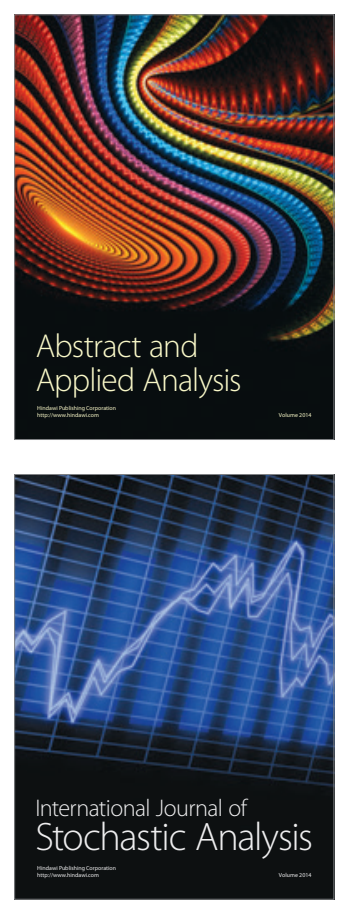

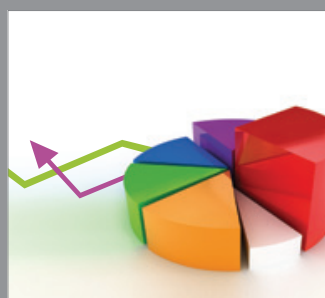

ournal of

Probability and Statistics

Promensencen
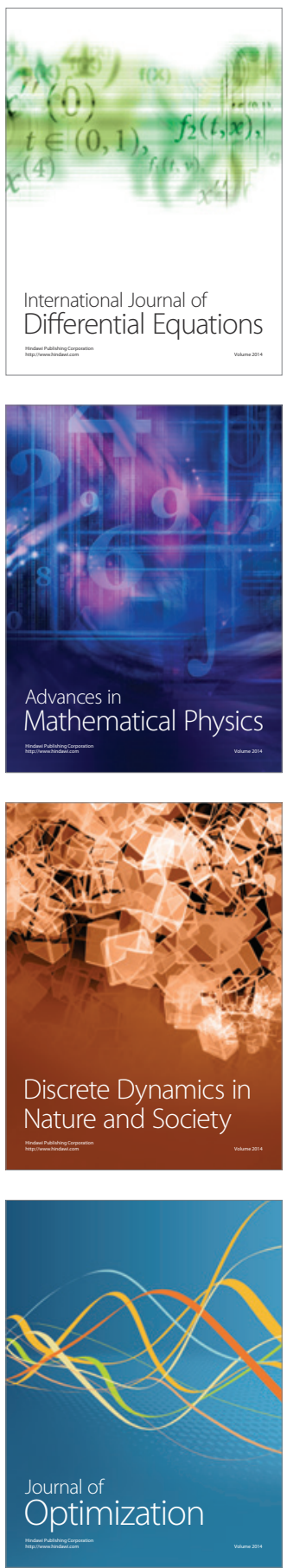